\title{
Stokes Approximation Solutions for Steep Standing Waves
}

\author{
Rodney J. Sobey \\ Department of Civil and Environmental Engineering, \\ Imperial College London, \\ London SW7 2AZ, UK \\ Phone: +44 (0)1289 303479, Fax: +44 (0)20 75945991 \\ E-mail: r.j.sobey@imperial.ac.uk
}

Coastal Engineering Journal, 54, (2), 2012

24 June 2012

\begin{abstract}
A hybrid analytical-numerical method for standing waves in water of any depth exactly satisfies the field equation, the bottom boundary condition, the periodic lateral boundary conditions and the mean water level constraint. The wave height and the kinematic and dynamic free surface boundary conditions are imposed numerically, as a problem in nonlinear optimisation. The algorithm is confirmed against an existing fifth-order analytical theory. The method extends the available predictive range for standing waves to near-limit waves in deep, transitional and shallow water. The limitations of the numerical method are clearly identified. The limit wave can not be predicted but near-limit extreme wave indicators for wave height, wave number and crest elevation are defined over the complete range of water depths.
\end{abstract}

Keywords Extreme wave kinematics, limit waves, long waves, nonlinear optimisation, numerical code, short waves, standing waves, unsteady flow.

\section{Introduction}

Standing waves appear in quite a wide variety of contexts in the natural and built environment. In the coastal environment, standing waves are a fundamental component of diffracted short wave fields about coastal structures and tidal long waves in estuaries. At a near-vertical sea wall, standing waves dominate the local flow pattern and define wave 
over-topping and pressures on the sea wall and the adjacent sea bed. Natural modes of enclosed or partially enclosed basins are standing wave modes.

In the built environment, there is interest in standing waves motions in closed vessels in motion, with applications in steel production and transport of various liquids and fuels in circular or rectangular tanks. Liquid oscillation in a storage tank, for example, may have a significant impact on the stability of a tanker, an aircraft or a rail car.

A final application is in the confirmation of numerical codes for free surface flows, where standing waves may provide a useful bench mark test.

The specific problem addressed herein is standing waves in a homogeneous, incompressible fluid (water or any other liquid) of constant depth that is periodic in a single horizontal direction $x$ and in time $t$. The literature on standing waves includes both analytical and numerical contributions; these will be reviewed in context below. The better of these theories are suitable for moderately steep standing waves in deep and perhaps deeptransitional water. Their suitability declines rapidly as the wave height increases above moderately steep in deep and transitional-deep water, and as the water depths decrease through transitional to shallow.

A hybrid analytical-numerical Stokes Approximation methodology is introduced with the intent of extending the range of secure applicability to extreme waves in deep water and to all wave heights in transitional and shallow water. The algorithm is demonstrated against an existing analytical theory. The limits of its applicability for waves of all heights and all water depths is extensively explored and documented.

\section{Problem Formulation}

Standing waves in horizontal dimension $x$, vertical dimension $z$ and time $t$ assume homogeneous, incompressible and irrotational flow such that the field equation is the Laplace equation

$$
\frac{\partial^{2} \phi}{\partial x^{2}}+\frac{\partial^{2} \phi}{\partial z^{2}}=0
$$

in which $\phi(x, z, t)$ is the velocity potential function. $x$ is measured in the plane of the Mean Water level (MWL) with $z$ directed upwards in opposition to the gravitational vector $\vec{g}$ (see Figure 1) The theoretical problem formulation is completed by

(i) the bottom boundary condition (BBC), requiring no flow through the horizontal bed,

$$
w(x, z=-h, t)=0
$$

in which $w=\partial \phi / \partial z$ is the vertical velocity,

(ii) the kinematic free surface boundary condition (KFSBC), requiring no flow through the instantaneous free surface,

$$
\frac{\partial \eta}{\partial t}+u \frac{\partial \eta}{\partial x}=w \quad \text { at } z=\eta(x, t)
$$

in which $u=\partial \phi / \partial x$ is the horizontal velocity, 


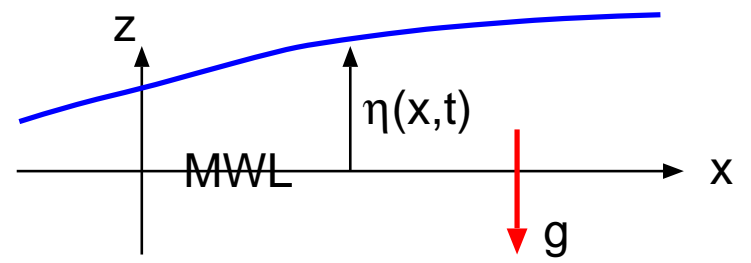

$z=-h$

Figure 1: Definition Sketch.

(iii) the dynamic free surface boundary condition (DFSBC), requiring zero atmospheric pressure at the instantaneous free surface,

$$
\frac{\partial \phi}{\partial t}+\frac{1}{2}\left(u^{2}+w^{2}\right)+g \eta=\bar{B} \quad \text { at } z=\eta(x, t)
$$

in which $\bar{B}$ is the Bernoulli constant,

(iv) the periodic lateral boundary conditions (PLBC), requiring periodicity in both $x$ and $t$

$$
\begin{aligned}
& \phi(x, z, t)=\phi(x+2 \pi / k, z, t)=\phi(x, z, t+2 \pi / \omega) \\
& \eta(x, z, t)=\eta(x+2 \pi / k, z, t)=\eta(x, z, t+2 \pi / \omega)
\end{aligned}
$$

in which $k$ is the wave number and $\omega$ is the wave frequency,

(v) the definition of wave height

$$
H=\eta(0,0)-\eta(L / 2,0)=\eta(0,0)-\eta(0, T / 2)
$$

in which $L=2 \pi / k$ is the wave length and $T=2 \pi / \omega$ is the wave period, and

(vi) the mass conservation requirement of an invariant mean water level (MWL)

$$
\int_{0}^{T} \int_{0}^{L} \eta(x, t) d x d t=0 .
$$

The nonlinearity of the standing wave problem is manifest through the free surface boundary conditions, Eqs. 3 and 4. 
Following analytical theories (Tadjbakhsh and Keller, 1960; Sobey, 2009) for Stokesstyle theories for standing waves, it is expected that the velocity potential function has the form

$$
\phi(x, z, t)=\sum_{j} \sum_{m} A_{j m} \frac{\cosh j k(h+z)}{\cosh j k h} \cos j k x \sin m \omega t
$$

and the water surface elevation the analogous form

$$
\eta(x, t)=\sum_{j} \sum_{m} b_{j m} \cos j k x \cos m \omega t
$$

$A_{j m}$ and $b_{j m}$ are constant coefficients.

For any integer $j$ and $m$, Equation 8 exactly satisfies the field equation (Equation 1 ) and the periodic lateral boundary conditions (Equation 5) for all $x, z$, and $t$, and also the bottom boundary condition (Equation 2) for all $x$ and $t$.

In Equations 8 and 9 , the coefficients $A_{00}$ and $b_{00}$ are recognised as the datums for $\phi$ and $\eta$ respectively. $b_{00}$ must be zero for the elevation datum plane assigned (see Figure 1) at the MWL. With this definition of $b_{00}$, the invariant MWL requirement (Eq. 7 ) is also exactly satisfied. The datum for $\phi$ is arbitrary, and $A_{00}$ is conveniently assigned as zero. Negative values of $j$ and $m$ do not provide a mathematically distinct contribution from their positive counterparts, and are omitted.

A significant feature of analytical theories for standing waves (Tadjbakhsh and Keller 1960, Section 3; Sobey 2009, Appendix 1) is zero values for the $A_{j m}$ and $b_{j m}$ coefficients when $j+m$ is an odd integer:

$$
A_{j m}=0, \quad b_{j m}=0 \quad \text { for } j+m=\text { odd integer }
$$

Half the unknown coefficients are expected to be non-zero. Equation 10 is explicitly adopted in the numerical problem formulation.

In any numerical method, summation must be truncated at a finite order. In principle, the $j$ and $m$ summations may be truncated at separate orders. But as the physical nature of the variation in both $x$ and $t$ is quite similar, there seems to be no specific advantage in assigning different summation limits. This was subsequently confirmed by numerical experiment. Accordingly, the Stokes approximation forms for $\phi$ and $\eta$ become

$$
\phi(x, z, t)=\sum_{j=0}^{M} \sum_{m=0}^{M} A_{j m} \frac{\cosh j k(h+z)}{\cosh j k h} \cos j k x \sin m \omega t \quad \text { for } j+m=\text { even }
$$

and

$$
\eta(x, t)=\sum_{j=0}^{M} \sum_{m=0}^{M} b_{j m} \cos j k x \cos m \omega t \text { for } j+m=\text { even }
$$

respectively, in which $M$ is the truncation order. 


\section{Numerical Solution}

The given information defining a standing wave problem will be

(i) the wave height $H$,

(ii) the water depth $h$,

(iii) the wave period $T$, and

(iv) the mass density $\rho$

Note the convenient specification of the wave period rather than the wave length, which has been the practice in some analytical theories (Penney and Price, 1952; Tadjbakhsh and Keller, 1960; Goda, 1967; Hsu et al., 1979) and some numerical theories (Vanden-Broeck and Schwartz, 1981; Tsai and Jeng, 1994) for standing waves. The choice of wave length rather that wave period among the given information involves some adjustments to the problem formulation; these are outlined in Appendix A.

The unknowns of the Stokes approximation problem are

(i) the wave number $k$,

(ii) the Bernoulli constant $\bar{B}$,

(iii) the non-zero $\phi$ coefficients $A_{j m}$, and

(iv) the non-zero $\eta$ coefficients $b_{j m}$.

At truncation order $M$, the unknowns number

$$
N_{\text {Unknowns }}= \begin{cases}(1+M)^{2} & \text { for } M \text { odd } \\ 1+(1+M)^{2} & \text { for } M \text { even }\end{cases}
$$

The equations available are

(i) the wave height

$$
\begin{aligned}
f_{H}\left(k, \bar{B}, A_{j m}, b_{j m}\right) & =0=\eta_{\text {Crest }}-\eta_{\text {Trough }}-H \\
& =\sum_{j=0}^{M} \sum_{m=0}^{M} b_{j m}-\sum_{j=0}^{M} \sum_{m=0}^{M} b_{j m}(-1)^{m}-H \quad \text { for } j+m=\text { even }
\end{aligned}
$$

(ii) the KFSBC at distributed water surface nodes $x_{i}, t_{n}$,

$$
f_{K}\left(k, \bar{B}, A_{j m}, b_{j m}\right)=0=\left.\frac{\partial \eta}{\partial t}\right|_{x_{i}, t_{n}}+\left.\left.u\right|_{x_{i}, \eta\left(x_{i}, t_{n}\right), t_{n}} \frac{\partial \eta}{\partial x}\right|_{x_{i}, t_{n}}-\left.w\right|_{x_{i}, \eta\left(x_{i}, t_{n}\right), t_{n}}
$$

and 
(iii) the DFSBC at distributed water surface nodes $x_{i}, t_{n}$

$$
\begin{aligned}
f_{D}\left(k, \bar{B}, A_{j m}, b_{j m}\right) & =0 \\
& =\left.\frac{\partial \phi}{\partial t}\right|_{x_{i}, \eta\left(x_{i}, t_{n}\right), t_{n}}+\left.\frac{1}{2}\left(u^{2}+w^{2}\right)\right|_{x_{i}, \eta\left(x_{i}, t_{n}\right), t_{n}}+\left.g \eta\right|_{x_{i}, t_{n}}-\bar{B}
\end{aligned}
$$

in which $u=\partial \phi / \partial x, w=\partial \phi / \partial z$ and also $\partial \phi / \partial t$ can be defined from the velocity potential function, Equation 11. The predictive equations are listed in Appendix B. Similarly, $\partial \eta / \partial t$ and $\partial \eta / \partial x$ can be defined from the water surface, Equation 12. The predictive equations are also listed in Appendix B

The space and time distribution of the water surface nodes $x_{i}, t_{n}$ can take advantage of some symmetries over the strict $0 \leq x \leq L, 0 \leq t \leq T$ solution domain. The sine and cosine terms in Equations 11 and 12 describe symmetry about the trough at $L / 2, T / 2$ :

$$
\phi(x, z, t)=\phi(2 \pi / k-x, z, 2 \pi / \omega-t), \quad \eta(x, t)=\eta(2 \pi / k-x, 2 \pi / \omega-t)
$$

Additionally, the Equation 10 constraint on non-zero values for the $A_{j m}$ and $b_{j m}$ coefficients in Equations 11 and 12 identify a further symmetry about $L / 4, T / 4$ :

$$
\phi(x, z, t)=-\phi(\pi / k-x, z, \pi / \omega-t), \quad \eta(x, t)=\eta(\pi / k-x, \pi / \omega-t)
$$

Accordingly, the unique extent of the solution domain is either $0 \leq x \leq L / 2,0 \leq t \leq T / 4$ (Vanden-Broeck and Schwartz, 1981) or $0 \leq x \leq L / 4,0 \leq t \leq T / 2$ (Tsai and Jeng, 1994; Okamura, 2003). The $\cos j k x$ and $\cos m \omega t$ or $\sin m \omega t$ variation in Equations 11 and 12 suggest that there is nothing to choose between these options. The former $(0 \leq x \leq L / 2$, $0 \leq t \leq T / 4)$ is adopted here.

The water surface nodes are distributed uniformly, at spatial resolution $x_{i}=i \Delta x$, $\Delta x=L / 2 I, i=0,1, \ldots I$, and temporal resolution $t_{n}=n \Delta t, \Delta t=T / 4 N, n=0,1, \ldots N$, with the expectation that $N$ is $I / 2$ (and rounded up for $I$ odd) The spatial and temporal distribution of water surface nodes need not be uniform, but the free surface boundary conditions must be satisfied for all $x$ and $t$. Physically, a uniform distribution of nodes must be preferred; a non-uniform distribution provides no apparent numerical advantage.

Equations 14, 15 and 16 are a system of $N_{\text {Equations }}=1+2(I+1)(N+1)$ nonlinear, implicit algebraic equations. Mathematical closure requires that $N_{\text {Equations }} \geq N_{\text {Unknowns }}$.

Physically, exact specification ties the spatial and temporal density of the nonlinear free surface boundary conditions to the assigned truncation order. Lower truncation order solutions are automatically associated with coarse spatial and temporal resolution of the nonlinear free surface boundary conditions, potentially compromising the global validity of the numerical solution.

Numerically, exact specification restricts the choice of numerical solution algorithm, omitting robust algorithms in nonlinear optimisation (see Press et al., 1992, Chapter 10). In addition, these multidimensional optimisation algorithms naturally accommodate overspecification $\left(N_{\text {Equations }}>N_{\text {Unknowns }}\right.$ ) of the problem, permitting the computational nodes $x_{i}, t_{n}$ to be assigned at a density that will physically reflect the spatial and temporal 
structure of the free surface and the free surface boundary conditions, Equations 15 and 16. A nodal density of $15 \times 8$ (144 nodes) over the region $0 \leq x \leq L / 2,0 \leq t \leq T / 4$ proved sufficient at truncation order $M=10$, providing 289 equations in 122 unknowns. At truncation order $M=15$, a nodal density of $20 \times 10$ (463 equations in 256 unknowns) proved suitable. In both cases, the computational resources demanded are not trivial. With attention to code vectorisation, the computational demands are manageable on currently available workstations.

The nonlinear optimisation algorithm adopted was the trust region method (Conn et al., 2000), based of the wave height (Equation 14), the KFSBC at the distributed water surface nodes (Equation 15), and the DFSBC at the distributed water surface nodes (Equation 16). The least squares objective function was

$$
\mathrm{O}\left(k, \bar{B}, A_{j m}, b_{j m}\right)=f_{H}^{2}+\frac{1}{(1+I)(1+N)} \sum_{i=0}^{I} \sum_{n=0}^{N}\left(f_{K}^{2}+f_{D}^{2}\right)
$$

to balance the respective contributions of the three physically distinct contributions to the equations.

To ensure the same prominence for the separate $f_{H}, f_{K}$ and $f_{D}$ contributions to the Equation 19 objective function, it is also numerically essential that each contribution has the same dimensions. This was achieved by non-dimensionalising every variable in the problem formulation by length and time scales that are

$$
\mathbb{L}=g / \omega^{2}, \quad \mathbb{T}=1 / \omega
$$

respectively.

The numerical success of nonlinear optimisation is critically dependent on the relative fidelity of the initial solution estimate. The very large number of unknowns in the present problem (typically 256) sharply focusses this problem. Three strategies were adopted, in sequential order.

(I) $k, \bar{B}$, and the coefficients $A_{j m}, b_{j m}$ through $A_{55}, b_{55}$ were assigned from an analytical Stokes-style theory to fifth order (Sobey, 2009). In the analytical theory, there are " $j m$ " contributions at each order $\mathrm{i}$, the coefficients being designated $\mathrm{A}_{\mathrm{ijm}}$ and $\mathrm{b}_{\mathrm{i} j m}$ respectively. The $A_{j m}$ and $b_{j m}$ were assigned as

$$
A_{j m}=\sum_{\mathrm{i}=1}^{5} \varepsilon^{\mathrm{i}} \mathrm{A}_{\mathrm{i} j m}, \quad b_{j m}=\sum_{\mathrm{i}=1}^{5} \varepsilon^{\mathrm{i}} \mathrm{b}_{\mathrm{i} j m}
$$

respectively, where $\varepsilon=k H / 2$. The initial estimates for $A_{j m}, b_{j m}$ coefficients beyond $A_{55}, b_{55}$ were zero.

(II) For extreme waves, the analytical theory to fifth order is not reliable and strategy I generally fails. The discussion in Sobey (2009) suggests that the analytical solution will not provide reasonable initial solution estimates for wave heights in excess of 
about $75 \%$ of the limit wave. For these wave conditions, successful solutions were often achieved with initial solution estimates provided by prior solutions at gradually increasing wave height (Rienecker and Fenton, 1981).

Assume a sequence of solutions $S(H)$, strictly $S(H ; h, T, \rho, M)$, where the parameters $h, T, \rho$ and $M$ are fixed. $S$ represents in turn elements of the solution vector $\left[k, \bar{B}, A_{j m}, b_{j m}\right]$. The strategy II initial solution estimate is

$$
S_{\text {Initial }}=S_{-1}
$$

where $S_{-1}$ is the solution vector at the next highest wave height $H_{-1}$ at which there was a successful solution.

(III) Strategy II in turn also sometimes fails for the more extreme wave conditions, especially in shallow water. The final strategy imposes a truncated Taylor series approximation. The strategy III initial solution estimate is

$$
S_{\text {Initial }}=S_{-1}+\frac{d S}{d H}\left(H-H_{-1}\right)
$$

where $d S / d H=\left(S_{-1}-S_{-2}\right) /\left(H_{-1}-H_{-2}\right)$. As before the "-1" subscript identifies the highest available successful solution. Similarly, "-2" identifies the next to highest successful solution.

The final possible difficulty is the rapid decline in the magnitude of the $A_{j m}$ and $b_{j m}$ coefficients as $j$ and $m$ increases, which may compromises the convergence criterion for the objective function. The suitability of scaling the $A_{j m}$ and $b_{j m}$ coefficients by $q^{j+m}$ during the numerical optimisation was investigated. A suitable constant $q$ factor is suggested by the fifth-order analytical theory. Extensive numerical evaluation of coefficient scaling was not encouraging. Identical solutions were achieved for small to moderate wave heights. At more extreme wave heights, scaling almost routinely provided a "no solution" result, in situations where no scaling $(q=1)$ provided a visually plausible and potentially physically plausible solution. Scaling was not routinely adopted.

Computations, at IEEE double precision, assigned convergence at a relative error in the non-dimensional objective function not exceeding $10^{-10}$. For objective functions at convergence typically less than $10^{-10}$ in absolute magnitude, this is a very demanding convergence criterion which imposed a very considerable computational penalty. The choice of a relative error not exceeding $10^{-10}$ was guided by preliminary numerical experiments.

Many aspects of the present algorithm were pioneered by Vanden-Broeck and Schwartz (1981). They rely on the third-order analytical solution of Tadjbakhsh and Keller (1960) to provide the ground truth, but this solution has been shown (Sobey, 2009, Table 1) to be accurate only to first order; this does appears to be implicitly recognised in their adoption of linear theory, rather than Tadjbakhsh and Keller (1960), to provide initial solution estimates. Their adopted $\left(x_{i}, t_{n}\right)$ field grid resolution at $6 \times 4$ is also much too sparse, but was no doubt dictated by available computational resources. 
The Tsai and Jeng (1994) algorithm adopts an analytical Stokes approximation for $\phi$ but not for $\eta$, which is discretely assigned as unknowns at the $\left(x_{i}, t_{n}\right)$ nodes of the field grid. This may be advantageous in seeking solutions that approach the limit waves. In application, the potential value has been compromised by an inappropriate use of the symmetry conditions, Equations 10 and 18. The adopted representation of $\eta$ is discrete and not an analytical Stokes approximation (like Equation 12). An expectation of $\eta$ symmetry about $(L / 4, T / 4)$ is not appropriate, and the imposition of the free surface boundary conditions over the truncated region $0 \leq x \leq L / 4,0 \leq t \leq T / 2$ must compromise the numerical solution. A second flaw is the incorrect restriction of the MWL constraint (Equation 7) to the same limited domain. Thirdly, they define convergence at an absolute error of $10^{-8}$, "achieved after three or four iterations". The present numerical experience suggests that this convergence criterion is not adequate, requiring a relative (not absolute) error of $10^{-10}$ (and very many more iterations). A final flaw is the imposition of post-numerical-solution smoothing of $\eta$ (Tsai and Jeng, 1994, Equation 15).

Okamura $(1997,2003)$ is a deep water only variation on Tsai and Jeng (1994). $\eta$ is calculated simultaneously from the DFSBC over a $0 \leq x \leq L / 4,0 \leq t \leq T / 2$ truncated domain. Wave height $H$ is not specified but rather the dimensionless crest acceleration $-D w /\left.D t\right|_{\mathrm{Cr}} / g$. Neither the MWL constraint nor the dispersion relationship are recognised. Near-limit solutions (at $-D w /\left.D t\right|_{\mathrm{Cr}} / g=0.9998$ ) are reported. $k$ and $\omega$ are not calculated, so that there are no predictions of field kinematics and no comparisons with analytical or alternative numerical solutions.

\section{Solution Kinematics}

Given a successful numerical solution, the complete field kinematics are available from the field predictions for the velocity potential function $\phi(x, z, t)$ (Equation 11).

The velocity components $u=\partial \phi / \partial x$ and $w=\partial \phi / \partial z$ were defined as part of the solution algorithm. The complete series summations are listed in Appendix B.

The acceleration components are

$$
\begin{aligned}
& \frac{D u}{D t}=\frac{\partial u}{\partial t}+u \frac{\partial u}{\partial x}+w \frac{\partial u}{\partial z} \\
& \frac{D w}{D t}=\frac{\partial w}{\partial t}+u \frac{\partial w}{\partial x}+w \frac{\partial w}{\partial z}
\end{aligned}
$$

The predictive equations for $\partial u / \partial t, \partial u / \partial x, \partial u / \partial z, \partial w / \partial t, \partial w / \partial x$ and $\partial w / \partial z$ are additionally listed in Appendix B.

Finally, the dynamic pressure is

$$
\frac{p_{d}}{\rho}=\bar{B}-\frac{\partial \phi}{\partial t}-\frac{1}{2}\left(u^{2}+w^{2}\right)
$$

The predictive equation for $\partial \phi / \partial t$ is listed in Appendix B. 


\section{Comparison of Analytical and Numerical Solutions}

From the experience of solutions for steady progressive waves, there is an expectation that Stokes Approximation solutions for standing waves will be strongly dependent of two dimensionless parameters:

- a depth parameter $\frac{\omega^{2} h}{g}$, and

- a height parameter $\frac{\omega^{2} H}{g}$

Note the implicit adoption of $g / \omega^{2}$ as the length scale and $1 / \omega$ as the time scale, which follows from the adoption of $T$ (and hence $\omega$ ) as a given parameter.

Depth influences are traditionally categorised as "wave deep" $\left(\omega^{2} h / g \gtrsim 2.5\right)$, "transitional" and "wave shallow" $\left(\omega^{2} h / g \lesssim 0.3\right)$. Height influences are categorised as "small" $\left(\omega^{2} H / g \rightarrow 0\right)$, "moderate height" and "near limit" $\left(\omega^{2} H / g \rightarrow \omega^{2} H_{\text {Limit }} / g\right)$ waves, in relational to the limit wave height $H_{\text {Limit }}$.

Literature estimates of limiting wave height for standing waves are sparse. For progressive waves, Williams (1985) provides tabulated predictions of the limit wave height for twenty-two $\omega^{2} h / g$ depths between 0.06 and 12.0. The theory is Stokes-style and computer extended to very high orders. A rational approximation to these tabulated predictions is

$$
\left.\frac{\omega^{2} H_{\text {Limit }}}{g}\right|_{\text {Progressive }}=c_{0} \tanh \frac{a_{1} p+a_{2} p^{2}+a_{3} p^{3}}{1+b_{1} p+b_{2} p^{2}}
$$

where $p=\omega^{2} h / g, a_{1}=0.7879, a_{2}=2.0064, a_{3}=-0.0962, b_{1}=3.2924, b_{2}=-0.2645$ and $c_{0}=1.0575$ has a maximum error of 0.0014 over the range of the tables.

For standing waves, the limit of validity for the Sobey (2009) analytical solution has been observed (see Sobey, 2009, Figure 10) to follow the trend of Equation 27 with dimensionless depth $\omega^{2} h / g$, but at approximately $89 \%$ of the limit wave height for progressive waves. Additionally, Mercer and Roberts (1992) predict a deep water $\left(\omega^{2} h / g=\infty\right)$ limit standing wave of $\omega^{2} H / g \approx 1.14$. This is approximately $108 \%$ of the limit wave height for progressive waves.

For preliminary reference purpose, both of these approximations for the limit wave height for standing waves will be cited, as

$$
\left.\left.\frac{\omega^{2} H_{\text {Limit }}}{g}\right|_{\text {Standing }} \sim[0.89 \mid 1.08] \frac{\omega^{2} H_{\text {Limit }}}{g}\right|_{\text {Progressive }}
$$

The $\left[c_{1} \mid c_{2}\right]$ notation has been adopted to denote alternate estimates of a parameter $c$.

The discussion will subsequently return ( $\S 8$ and $\S 9)$ to a somewhat more satisfactory definition of near-limit conditions for standing waves. 


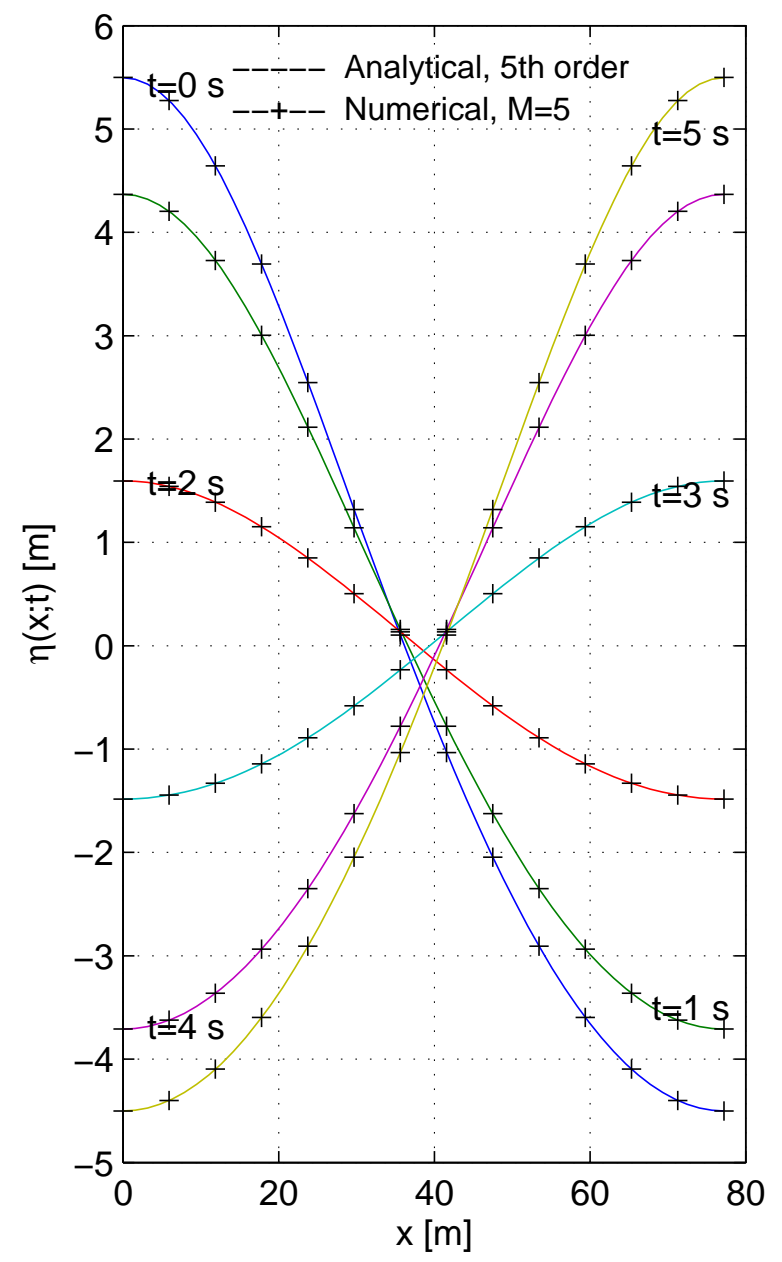

(a) $\eta(x ; t)[m]$

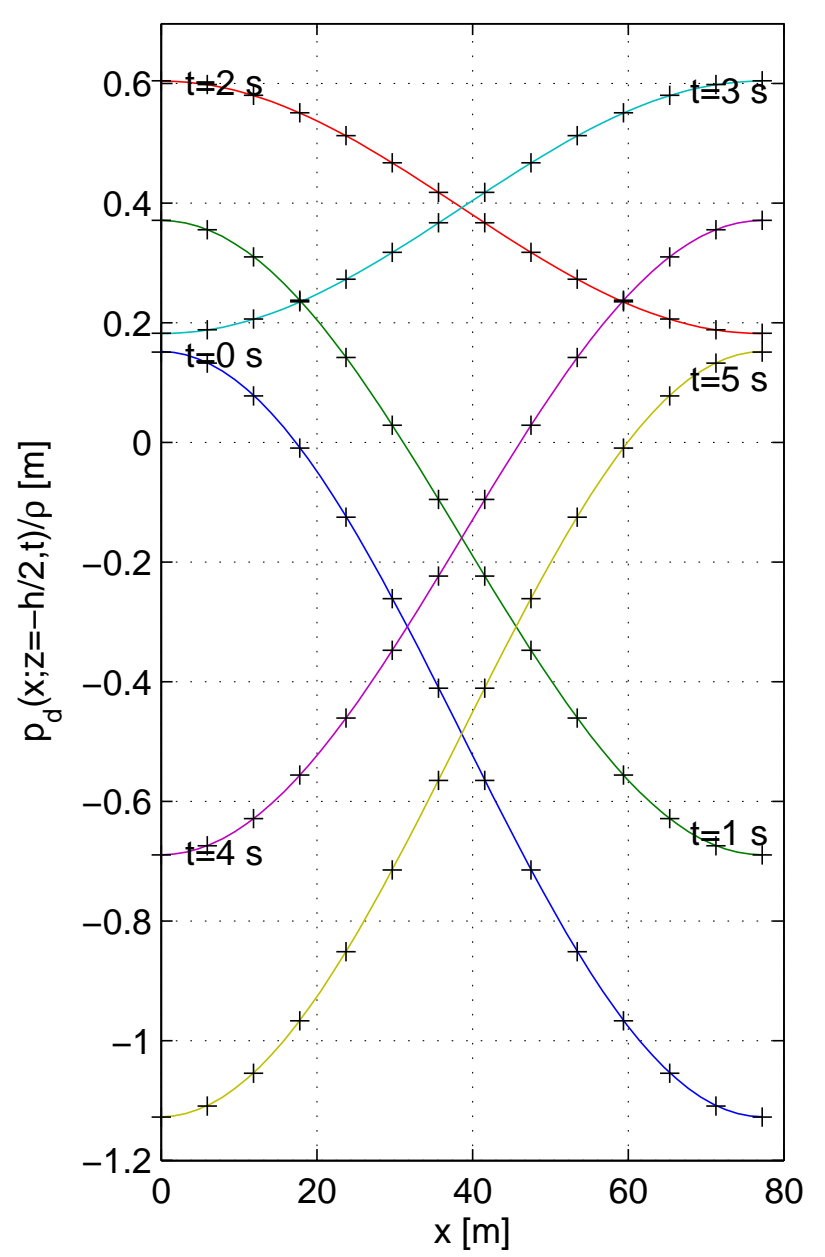

(b) $p_{d}(x ; z=-h / 2, t) / \rho[m]$

Figure 2: $\eta$ and $p_{d}$ Evolution for a Deep Water Wave. $T=10 \mathrm{~s}, h=100 \mathrm{~m}, H=10 \mathrm{~m}$

Numerical Solution Confirmation. An immediate check on the veracity of the numerical problem formulation and algorithm coding is provided by the Sobey (2009) analytical solution to fifth order.

A specific example solution therein (see Sobey, 2009, Figures 2-6) has wave period $10 \mathrm{~s}$, water depth $100 \mathrm{~m}$ and wave height $10 \mathrm{~m}$. The Equation 27 based limit wave approximations are $[23.3 \mid 28.2] \mathrm{m}$. These are "wave deep" $\left(\omega^{2} h / g=4.02\right)$ and "moderate height" $\left(\omega^{2} H / g=0.40\right)$ conditions, and well within the region of certain applicability of the analytical theory.

Attention is directed to the water surface elevation $\eta(x ; t)$ and the dynamic pressure $p_{d}(x ; z=-h / 2, t)$ at times $t=0,1,2,3,4,5$ s over a half-cycle. Figure 2 shows the 5 th order analytical solution together with numerical Stokes approximation solutions for the directly comparable truncation order $M$ of 5 . Agreement here is visually excellent between the 5 th 


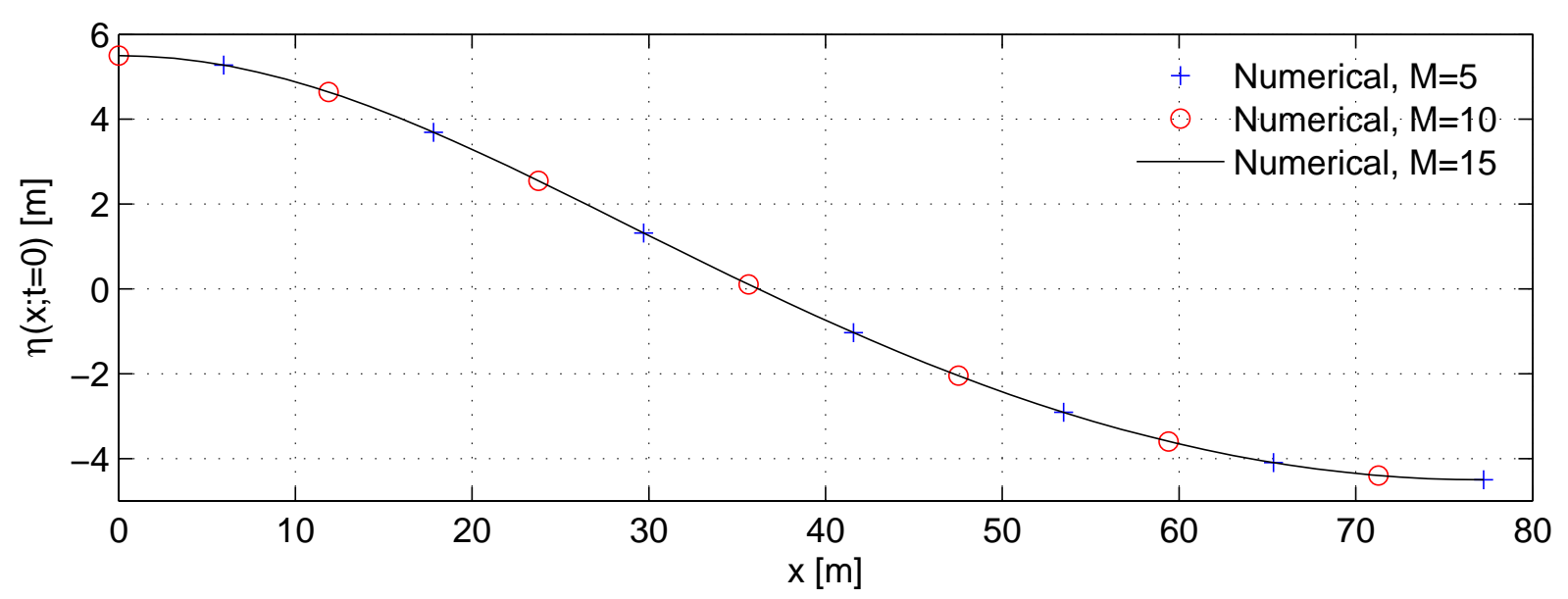

Figure 3: $\eta(x ; t=0)$ at Different Truncation Orders. Moderate Deep Water Wave. $T=10$ $\mathrm{s}, h=100 \mathrm{~m}, H=10 \mathrm{~m}$

order analytical and the $M=5$ numerical solutions.

The impact of truncation order $M$ at these wave conditions is explored in Figure 3 for $M=5,10$ and 15 . For clarity, only the odd(even) numbered numerical nodes are plotted for the $M=5(10)$ numerical solutions.

Any change from $M=5$ to 10 and 15 is visually insignificant. This was the expected result, being also the trend predicted for steady progressive waves (Sobey, 1989) of moderate height in deep water.

The potential value of the numerical Stokes approximation theory is in extending the range of viable solutions to both "wave shallow" $\left(\omega^{2} h / g \rightarrow 0\right)$ and "extreme height" $\left(\omega^{2} H / g \rightarrow \omega^{2} H_{\text {Limit }} / g\right)$ conditions. Two waves are defined to explore the numerical solution potential. The first is a near-limit height wave in deep water, the second a wave of moderate height in shallow water.

Near-limit Height Wave in Deep Water. For this problem, the wave period is $10 \mathrm{~s}$, the water depth is $100 \mathrm{~m}$ and wave height is $25 \mathrm{~m} . \omega^{2} h / g$ is 4.02 and $\omega^{2} H / g$ is 1.01 . The Equation 27 based limit wave approximations remain [23.3|28.2] m. Numerical solutions for $M=5,10$ and 15 are again compared in Figure 4, together with the analytical solution. That there is no visual difference among the numerical predictions demonstrates the values of the Stokes Approximation solution in extending the range of viable solutions to very steep waves in deep water. The analytical solution has minor oscillations about the consensus numerical profile, an indication of its extension beyond its domain of applicability.

Moderate Height Wave in Shallow Water. For this problem, the wave period is 10 $\mathrm{s}$, the water depth is $5 \mathrm{~m}$ and wave height is $2.5 \mathrm{~m}$. $\omega^{2} h / g$ is 0.20 and $\omega^{2} \mathrm{H} / \mathrm{g}$ is 0.10 . The Equation 27 based limit wave approximations are [3.4|4.1] $\mathrm{m}$. The predicted water 


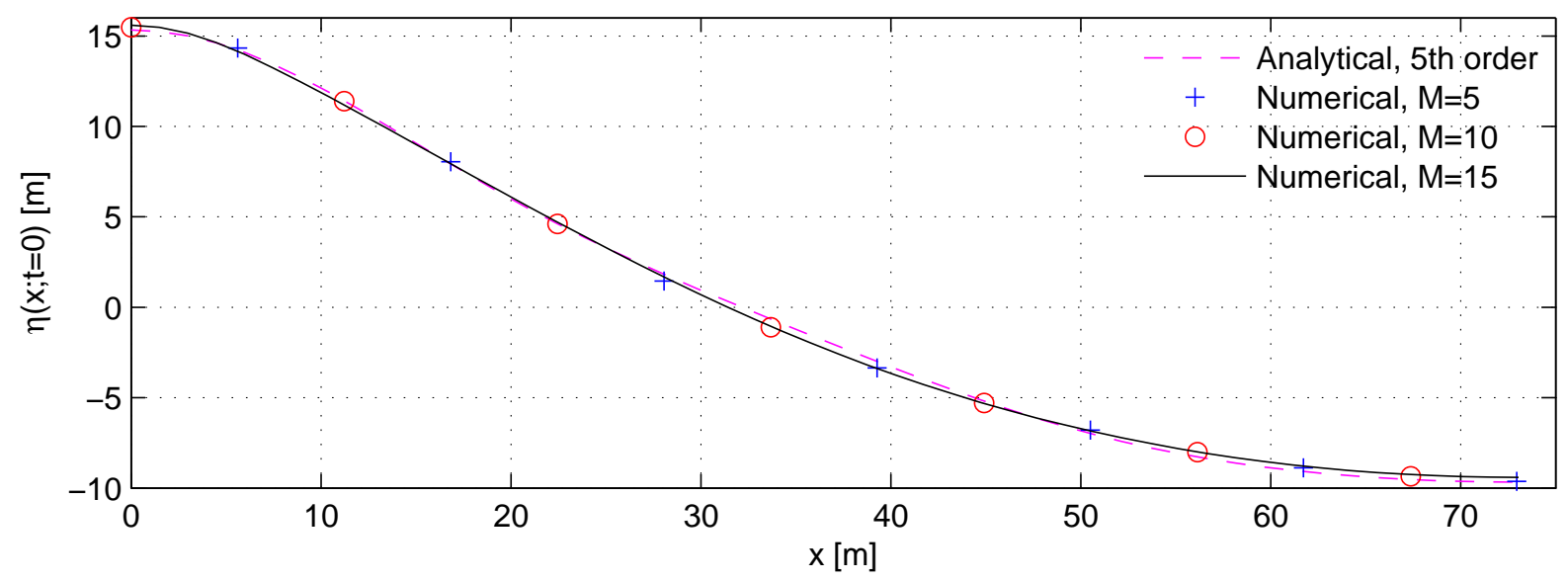

Figure 4: $\eta(x ; t=0)$ at Different Truncation Orders. Near-Limit Deep Water Wave. $T=10$ $\mathrm{s}, h=100 \mathrm{~m}, H=25 \mathrm{~m}$

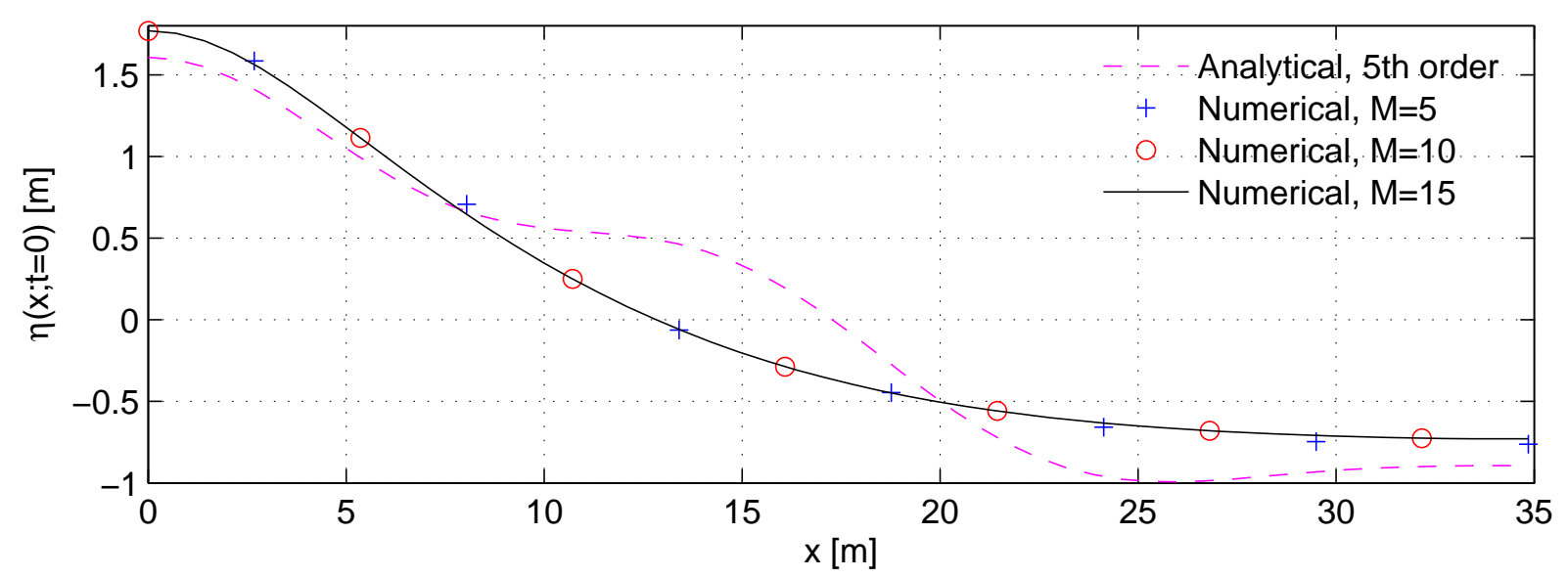

Figure 5: A Moderate Height Wave in Shallow Water. $T=10 \mathrm{~s}, h=5 \mathrm{~m}, H=2.5 \mathrm{~m}$

surface profiles at $t=0$ are shown in Figure 5 , for the 5 th order analytical solution and numerical Stokes approximation at $M=5,10$ and 15. It is immediately clear from the major profile curvature reversals that the 5 th order analytical solution is beyond its domain of applicability. The $M=10$ numerical result does seem to be a physically acceptable prediction, especially as it is confirmed by the $M=15$ solution. The $M=5$ numerical solution is marginally steeper, suggesting that a $M=5$ truncation order is insufficient in shallow water.

The time evolution of the predictions for the water surface and dynamic pressure profiles at half-depth are shown in Figure 6, where the $M=5$ and 15 predictions have been excluded. The $M=10$ numerical predictions remain physically plausible. 


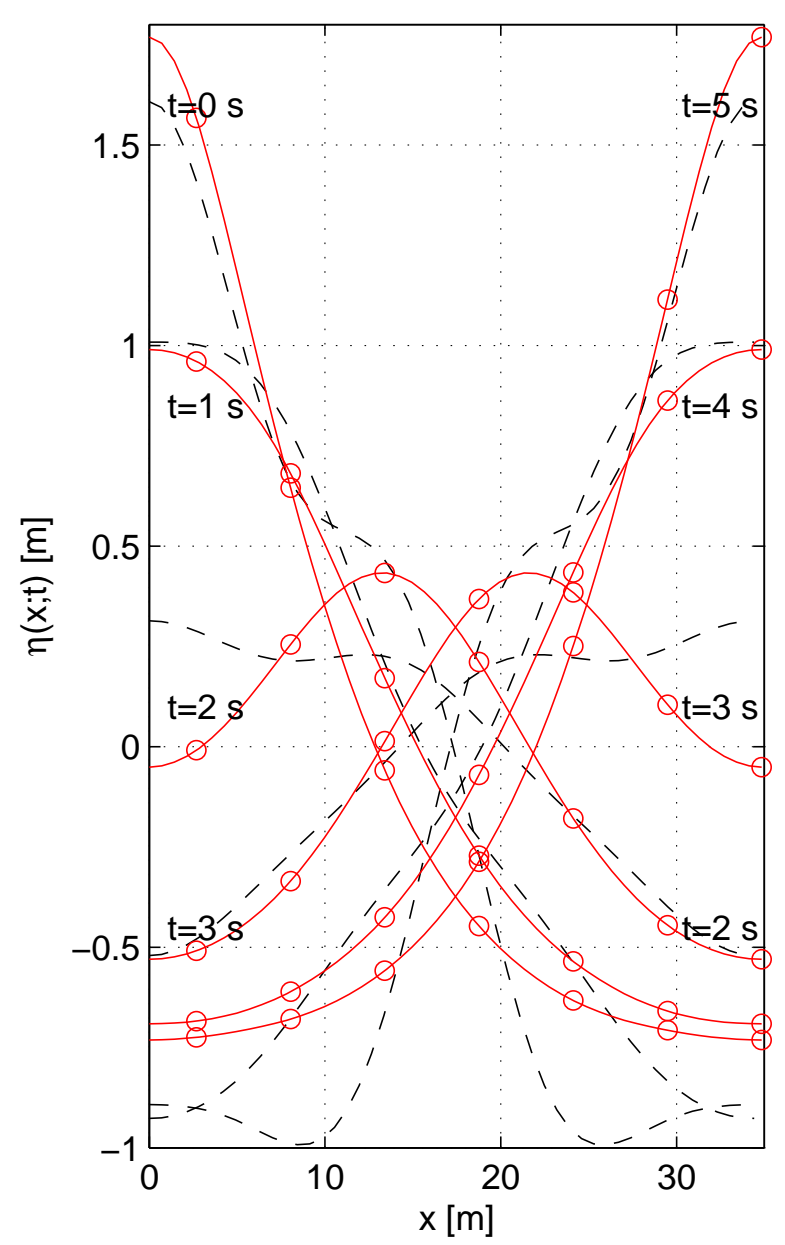

(a) $\eta(x ; t)[m]$

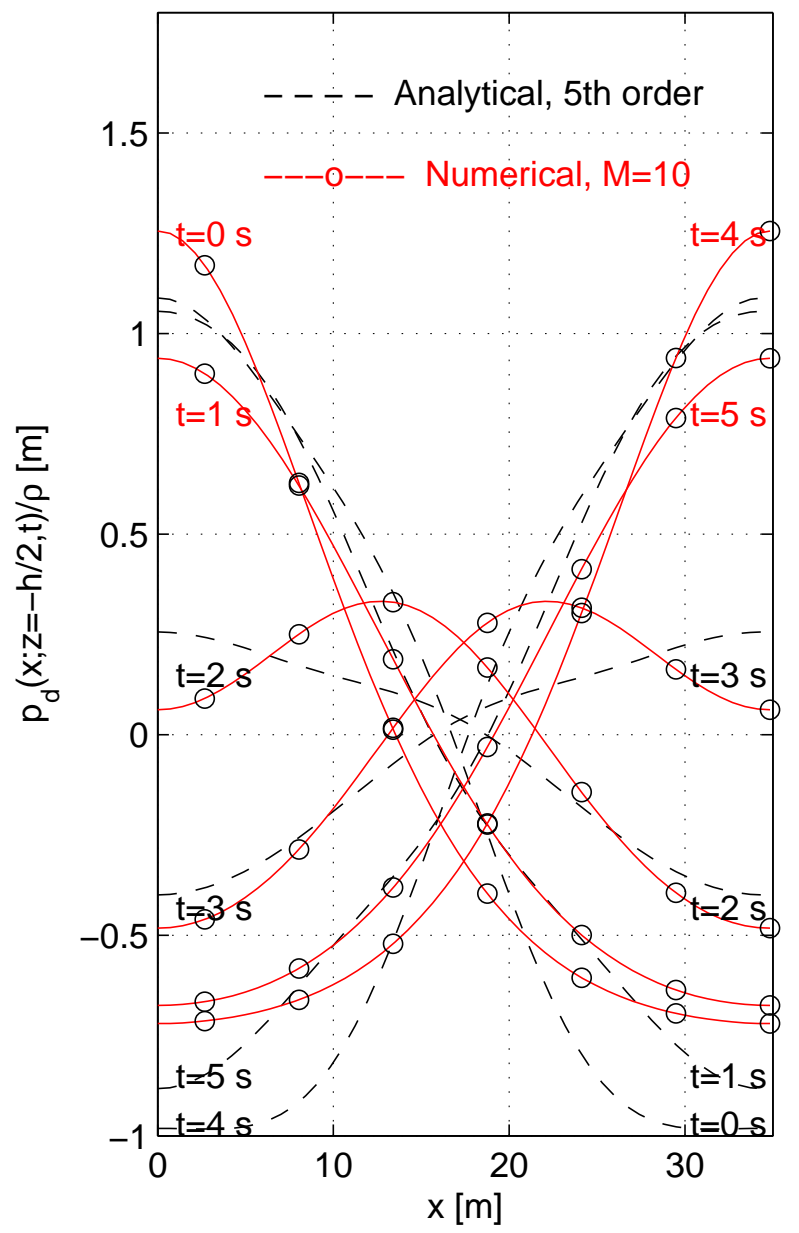

(b) $p_{d}(x ; z=-h / 2, t) / \rho[m]$

Figure 6: $\eta$ and $p_{d}$ Evolution for a Moderate Height Wave in Shallow Water. $T=10 \mathrm{~s}, h=5$ $\mathrm{m}, H=2.5 \mathrm{~m}$

\section{What is a Successful Numerical Solution?}

The preceding discussion raises the issue of what is and what is not a successful solution.

No numerical convergence would seem to imply no successful solution, but this may just be an indication that the numerical solution algorithm has not located the neighbourhood of the unique physical solution. The very significant impact of the initial solution estimate in locating the physical solution neighbourhood has been acknowledged in the preceding description of the numerical solution algorithm. Strategies I though III are sequentially attempted before a wave condition $(h, T$ and $H)$ is abandoned.

But an apparently successful numerical solution is not necessarily an appropriate physical solution. Careful exploration of all aspects of the predicted solution is essential. Extensive numerical experiment has suggested a focus on the following features: 


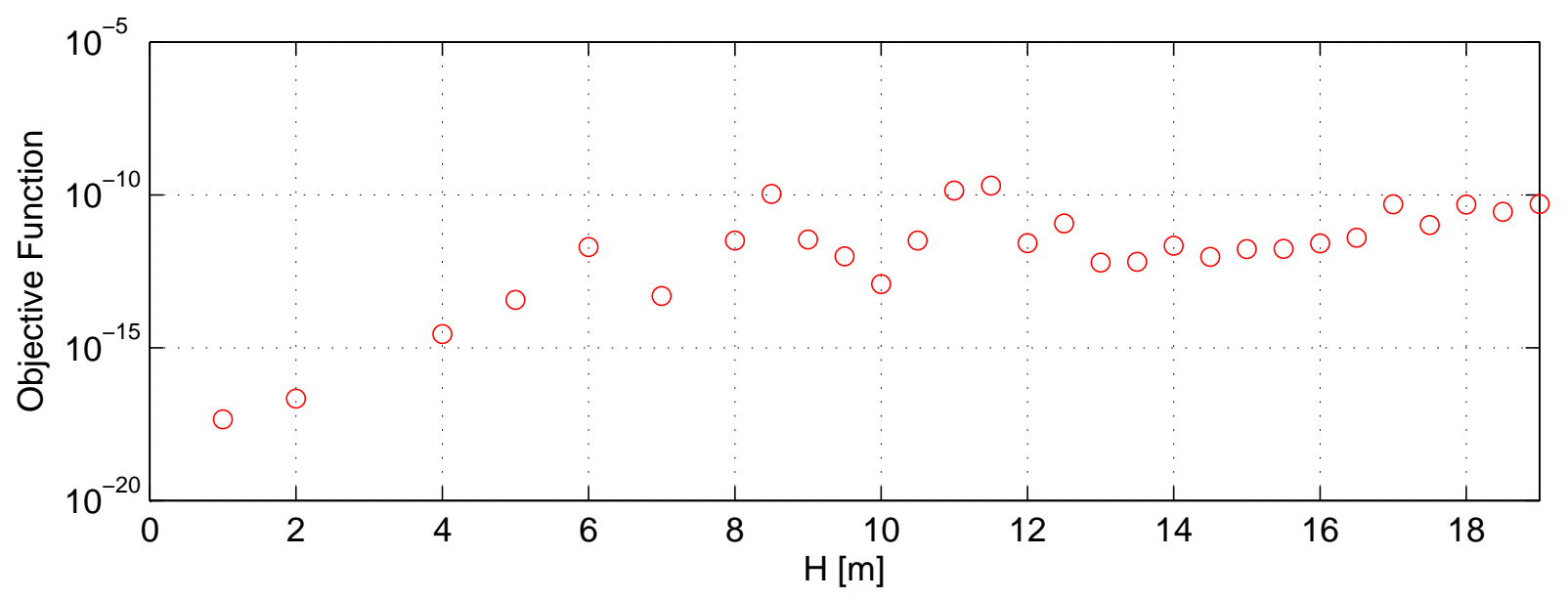

Figure 7: Objective Function evolution for $h=20 \mathrm{~m}, T=10 \mathrm{~s}, M=15$.

(A) Convergence to a sufficiently small non-dimensional objective function.

This is an obvious and very essential first check. A failure of the numerical algorithm to converge is generally signalled very clearly, typically by a NaN (Not-a-Number) result for one or more of the Equation 15 or 16 free surface boundary conditions at the distributed water surface nodes.

Where numerical convergence is achieved however, it does not necessarily provided compelling evidence to either accept or reject a numerical solution.

Figure 7 shows the evolution of the Equation 19 objective function at convergence for a "wave transitional" context. The magnitude of the objective function generally increases with increasing wave height, but it is still of order $10^{-10}$ for the most extreme solution.

(B) A monotonically evolving water surface profile with $x$ for a fixed $t$, or with $t$ for a fixed $x$.

Perusal of the complete $\eta(x, t)$ surface proved to be a necessarily routine practice. A focus on the $\eta(x, t=0)$ and $\eta(x=0, t)$ profiles would be a minimum requirement.

The expectation of a monotonically evolving water surface profile with $x$ or $t$ is observational and physical. It is not imposed through the numerical solution algorithm, Equations 11, 12 and 14 through 19.

A common early indication of an unsuccessful solution is the appearance of solution oscillations near the trough. Such numerical solutions do not violate the present numerical problem formulation, but they do assert that the numerical solution has not identified a physical solution neighbourhood.

(C) The field predictions for the dynamic pressure $p_{d}$. 
Some quite distinctive aspects of the $p_{d}(x, z, t)$ solution field have been identified in nonlinear analytical solutions (Goda, 1967; Sobey, 2009). In particular, at any elevation

(i) The profile asymmetry is reversed, with the maximum amplitude associated with the wave trough rather than the wave crest.

(ii) The time-histories nearer the crest and trough are no longer monotonic but exhibit a double profile curvature reversal.

(iii) The maximum amplitudes are no longer at $x=0$ and $L / 2, t=0$ and $T / 2$, but have moved closer to the quarter wavelength $x=L / 4, t=T / 4$ locations.

The dynamic pressure provides an excellent assessment of a high order solution precisely because of the implicit magnification of higher order contributions to the solution through the $u^{2}$ and $w^{2}$ terms. A generally similar message is also provided by the acceleration predictions.

A focus on the time histories $p_{d}(t ; x=0, z=Z) / \rho$ has proved revealing; useful elevations for $Z$ are the trough elevation $Z=\eta_{\operatorname{Tr}}$ and the half-depth $Z=-h / 2$.

Dynamic pressure frequently provides the necessary confirmation of the judgement already suggested by feature (B).

\section{Response Patterns for Steepening Waves}

The major disappointment of the fifth-order analytical theory for standing waves was its progressive failure (see Sobey, 2009, Figure 9) beyond moderately steep $\left(\omega^{2} H / g \gtrsim 0.9\right)$ conditions in deep water $\left(\omega^{2} h / g \lesssim 2.5\right)$. This was expected of a Stokes-style theory. Among local solution variables, the relative crest elevation, $\eta_{C r} / H$, reaches a predicted maximum and the vertical acceleration at the crest $(D w / D t)_{C r}$ reaches a predicted minimum. These predictions are not physically appropriate. Additionally, the predictive potential of the analytical solution deteriorates rapidly as the dimensional water depth $\omega^{2} h / g$ shallows.

Predictions from the numerical Stokes Approximation theory are rather more compelling. The wave number, the relative crest elevation and the vertical acceleration at the crest evolve smoothly with increasing wave height, following the trends initially established by the analytical theory to rather larger wave heights. With increasing wave height, clear indications of physically unacceptable predictions eventually become apparent, especially with the vertical acceleration.

Solution evolution predictions at depths of $100 \mathrm{~m}, 20 \mathrm{~m}$ and $5 \mathrm{~m}$ are explored below; the period was constant at $10 \mathrm{~s}$. The truncation order was set at $M=15$, in anticipation of the subsequent discussion of limit waves. At each depth, a data base of Stokes Approximation solutions was established, starting at very small wave heights and extending to the largest height where a visually acceptable solution could still be achieved. The solution evolution is presented for the wave number $k$, the relative crest elevation, $\eta_{C r} / H$, and the relative vertical acceleration at the crest $(D w / D t)_{C r} / g$. 

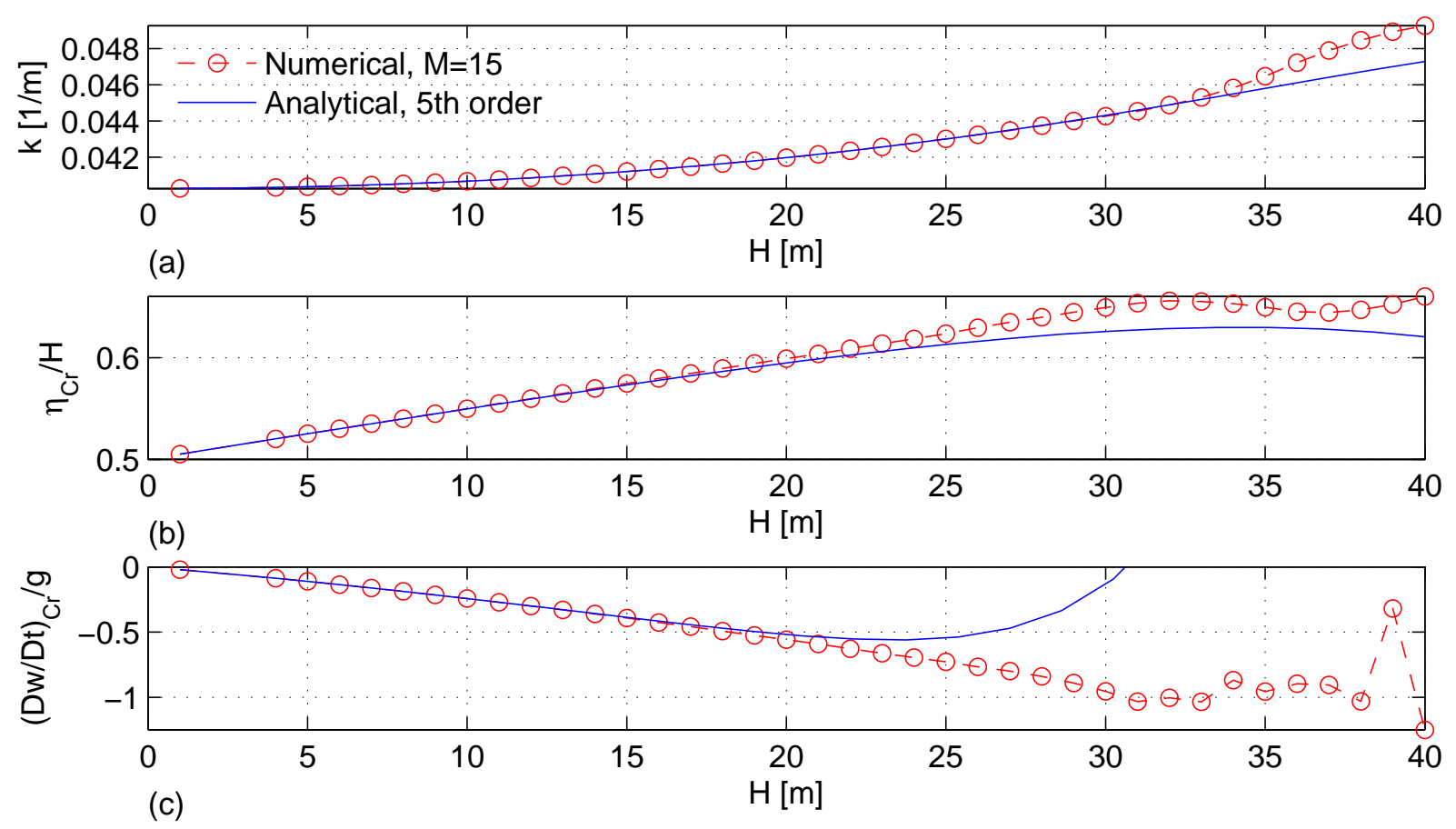

Figure 8: Solution evolution for $h=100 \mathrm{~m}, T=10 \mathrm{~s}, M=15$.

Wave deep response. As a "wave deep" illustration, a sequence of numerical solutions were established at depth $h=100 \mathrm{~m}$, period $T=10 \mathrm{~s}$ and truncation order $M=15$. The Equation 27 based approximations for the limit wave are [23.3|28.2] $\mathrm{m}$. The wave height $H$ was progressively increased while visually successful solution could still be obtained. The numerical predictions are shown in Figure 8 as the "o" markers, together with the corresponding predictions from the fifth-order analytical theory.

Part (a) shows the evolution of the wave number $k$, part (b) the crest elevation $\eta_{\text {Cr }}$ and part (c) the vertical acceleration $(D w / D t)_{\mathrm{Cr}}$ at the crest. Each evolves largely as expected. The wave number increases with wave height as the wave steepens. At $H=0$ the wave number is $k_{\text {Airy }}$, as predicted by the linear standing wave theory dispersion relationship,

$$
\omega^{2}=g k \tanh k h
$$

The crest profile sharpens as the height increases; for very small $H$, the crest elevation is $\eta_{\mathrm{Cr}}=H / 2$ as in the linear standing wave theory. The $(D w / D t)_{\mathrm{Cr}}$ predictions evolve from zero toward $-g$ as the height increases; the zero value at $H=0$ again corresponds with the linear standing wave theory. $k, \eta_{\mathrm{Cr}}$ and $(D w / D t)_{\mathrm{Cr}}$ evolve smoothly with increasing $H$, separating from the analytical predictions at $H>30 \mathrm{~m}$ for wave number and at $H \approx 20 \mathrm{~m}$ for crest elevation and vertical acceleration.

Physically unacceptable numerical solutions appear to be announced by the trend deviation around $H \approx 30 \mathrm{~m}$ for all predictions. The erratic response in the vertical acceleration 

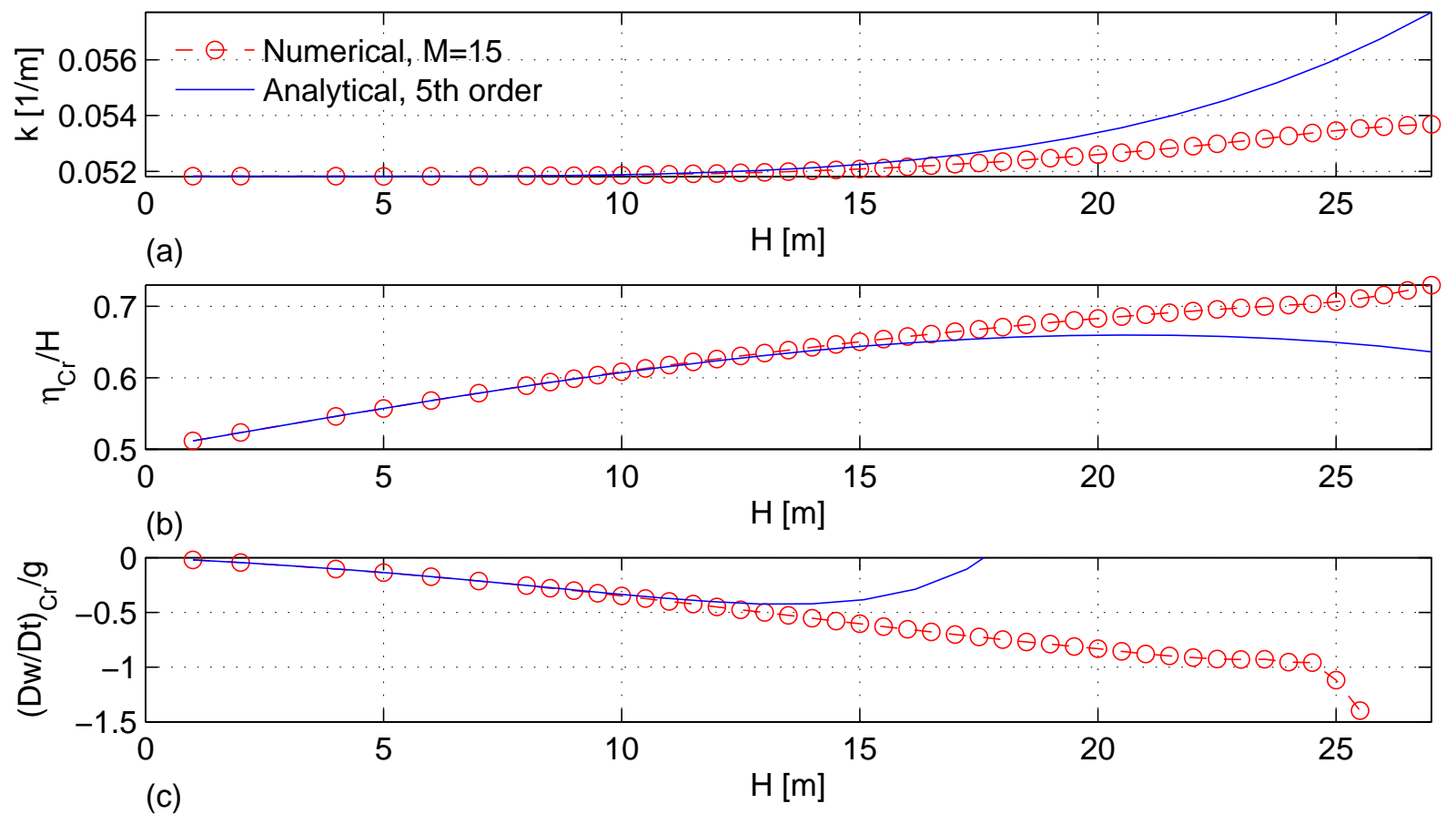

Figure 9: Solution evolution for $h=20 \mathrm{~m}, T=10 \mathrm{~s}, M=15$.

confirms that wave heights of this magnitude will provide physically unacceptable solutions.

Both the analytical theory and the numerical Stokes Approximation theory assume a smooth and continuous crest profile. The limit wave is expected (Penney and Price, 1952) to be very sharp and perhaps discontinuous at the crest. Neither theory can represent a discontinuous crest profile, so that the highest credible wave height solution will be short of the limit wave. There is detailed discussion of this issue in the following section, $\S 8$.

Transitional depth response. As a "transitional depth" illustration, a sequence of numerical solutions were established at depth $h=20 \mathrm{~m}$, period $T=10 \mathrm{~s}$ and truncation order $M=15$. The Equation 27 based approximations for the limit wave are [11.6|14.0] $\mathrm{m}$.

The wave height $H$ was progressively increased while visually successful solution could still be obtained. The numerical predictions are shown in Figure 9, together with the corresponding predictions from the fifth-order analytical theory.

The value of the numerical Stokes Approximation solution is immediately apparent in Figures 9b and especially 9c. The analytical predictions in Figure 9b of a maximum for relative crest elevation and in Figure $9 \mathrm{c}$ of a minimum in vertical acceleration strongly suggest that the analytical theory has been extended beyond its domain of applicability (as anticipated in Sobey, 2009, Figure 9). Parts b and c collectively suggest that the analytical theory remains appropriate up to wave heights of order $10 \mathrm{~m}$ for $h=20 \mathrm{~m}$ and $T=10 \mathrm{~s}$.

The numerical Stokes Approximation predictions appear reliable to wave heights of 

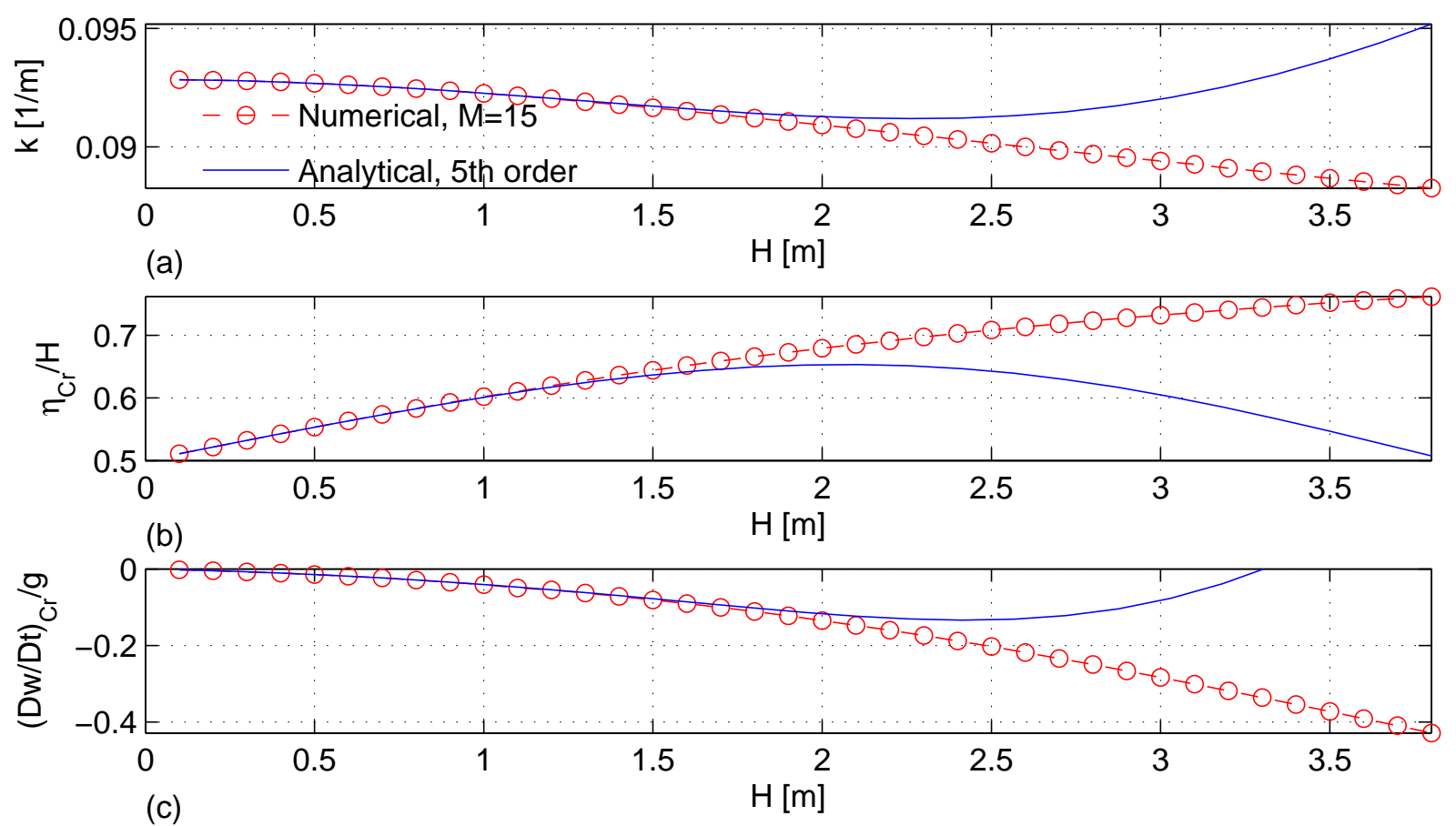

Figure 10: Solution evolution for $h=5 \mathrm{~m}, T=10 \mathrm{~s}, M=15$.

order $20 \mathrm{~m}$. The trends generally shown in Figure 9 are monotonic and follow those established by the analytical solution at lower wave heights.

Again, the largest visually successful solution exceeds the Equation 27 based approximations of $[11.6 \mid 14.0] \mathrm{m}$, and the numerical $D w / D t$ predictions decrease beyond $-g$.

Wave shallow response. As a "wave shallow" illustration, a sequence of numerical solutions were established at depth $h=5 \mathrm{~m}$, period $T=10 \mathrm{~s}$ and truncation order $M=10$. The numerical and corresponding analytical predictions are shown in Figure 10.

The trend, of declining applicability of the analytical theory and increasing utility for the numerical Stokes Approximation theory as the wave height increases, continues. The analytical theory has credibility only for very small wave heights, perhaps to $H \approx 1.5 \mathrm{~m}$ in this case. The numerical theory apparently extends this applicability to $H \approx 3.5 \mathrm{~m}$, in the neighbourhood of the Equation 27 based approximations of [3.4|4.1] m.

The Stokes Approximation theory remains a Stokes-style theory and its applicability is not expected to be strong in shallow water. This is underlined by the vertical acceleration at $H=3.5 \mathrm{~m}$ (Figure 10c), which has not even reached $-g / 2$.

The wave number is now monotonically decreasing with wave height (Figure 10a), reversing the trend predicted in transitional and deep water. This trend is repeated at progressively smaller depths, and is consistent with Vanden-Broeck and Schwartz (1981, Figure 1). Progressive waves, in comparison, have the wave number decreasing monotoni- 


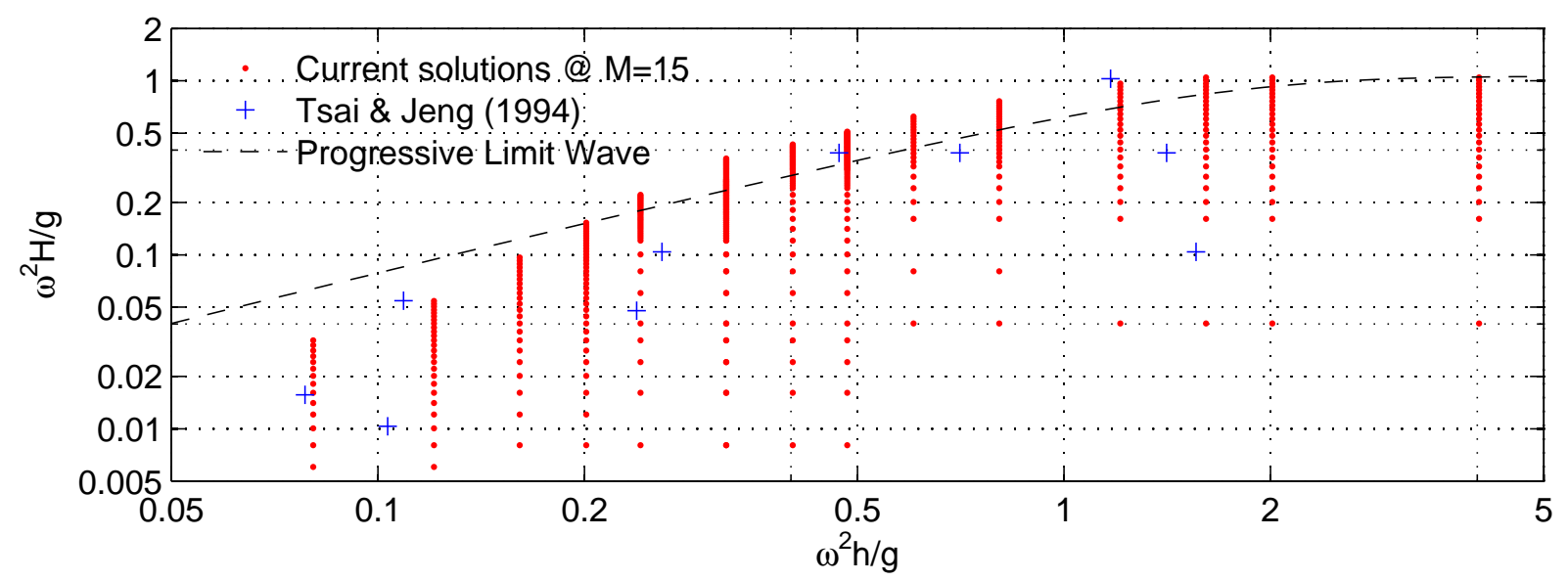

Figure 11: Data Base of Viable Standing Wave Solutions.

cally with increasing wave height over the complete range of depths (Sobey, 2012, Figures $3 \mathrm{a}$ and $6 \mathrm{a})$.

Figures 8 through 10 collectively suggest that applicability of the numerical Stokes Approximation theory at appropriate order is significantly more extensive than the analytical theory. Nevertheless, the limiting trends remain those suggested by the analytical theory. The validity is strong in deep water and declines as the water shallows. Steep waves are easily accommodated in deep water, but less so as the water shallows.

\section{Physically Appropriate Solutions}

As acknowledged above, the numerical Stokes Approximation theory can not provide credible solutions for the limit standing wave. The theory assumes a smooth and continuous crest profile. The limit wave is expected (Penney and Price, 1952) to be very sharp and perhaps discontinuous at the crest. The theory can not represent a discontinuous crest profile, so that the highest credible wave height solution will be short of the limit wave.

Nevertheless, it is apparent from Figures 8 through 10 that viable solutions are available for quite extreme waves, especially in deep water where these solutions reach to the neighbourhood of the limit wave. While credible solutions for the kinematics in limit standing waves are not feasible from this Stokes Approximation theory, trend extrapolation might potentially provide estimates of $H_{\text {Limit }}$, the limit height of standing waves.

In further pursuit of this objective, the sequence of solutions introduced in Figures 8 through 10 was extended to depths $h$ of $50,40,30,15,12,10,8,7,6,4,3$ and $2 \mathrm{~m}$ at period $T=10 \mathrm{~s}$. This data base of successful solutions is represented in Figure 11 as $\omega^{2} H / g=f\left(\omega^{2} h / g\right)$, following the introduction in $\S 5$ of $g / \omega^{2}$ as the length scale and $1 / \omega$ as the time scale. Published numerical solutions of Tsai and Jeng (1994) are also shown as " +'. Unfortunately, they do not provide $\omega$, so that the "+" points involve some interpretation 
that uses the present Stokes Approximation solutions. Neither do they provide sufficient information for comparison of predicted kinematics with the present Stokes Approximation solutions.

As a useful datum for comparison, Figure 11 also includes the Equation 27 rational approximation to the limit wave height for progressive waves. Note that viable solutions for standing waves reach or exceed the progressive limit wave over the entire transitional to deep water region. Only in shallow water do viable standing wave solutions not reach the progressive limit wave; this will subsequently be related to the extension of the present Stokes-style theory beyond its optimum context.

The Figure 11 data base of solutions from the present Stokes Approximation theory may possibly be used to estimate the limit wave height. But it is necessary first to distinguish between a visually successful solution and a physically appropriate solution.

Penney and Price (1952) argue that the limit standing wave

(1) cannot withstand tension at the water surface

As $p=0$ at the water surface from the dynamic free surface boundary condition, the pressure just below the free surface must be zero or positive:

$$
\frac{\partial p}{\partial z} \leq 0 \quad \text { at } z=\eta(x, t)
$$

(2) corresponds to instantaneously stationary conditions where

$$
u(x, z, t)=w(x, z, t) \equiv 0
$$

(3) has a profile discontinuity at the crest with an included angle of $\pi / 2$.

As this remains potential flow, the vertical momentum (Euler) equation is

$$
\frac{D w}{D t}=-g-\frac{1}{\rho} \frac{\partial p}{\partial z}
$$

Using Equation 31 for a limit wave, Equation 32 becomes

$$
\frac{\partial w}{\partial t}=-g-\frac{1}{\rho} \frac{\partial p}{\partial z}
$$

Further, using Equation 30 at the water surface

$$
\frac{\partial w}{\partial t} \geq-g \quad \text { at } z=\eta(x, t)
$$

which becomes the crest condition

$$
\left.\frac{D w}{D t}\right|_{\mathrm{Cr}}=\left.\frac{\partial w}{\partial t}\right|_{\mathrm{Cr}}=-g
$$




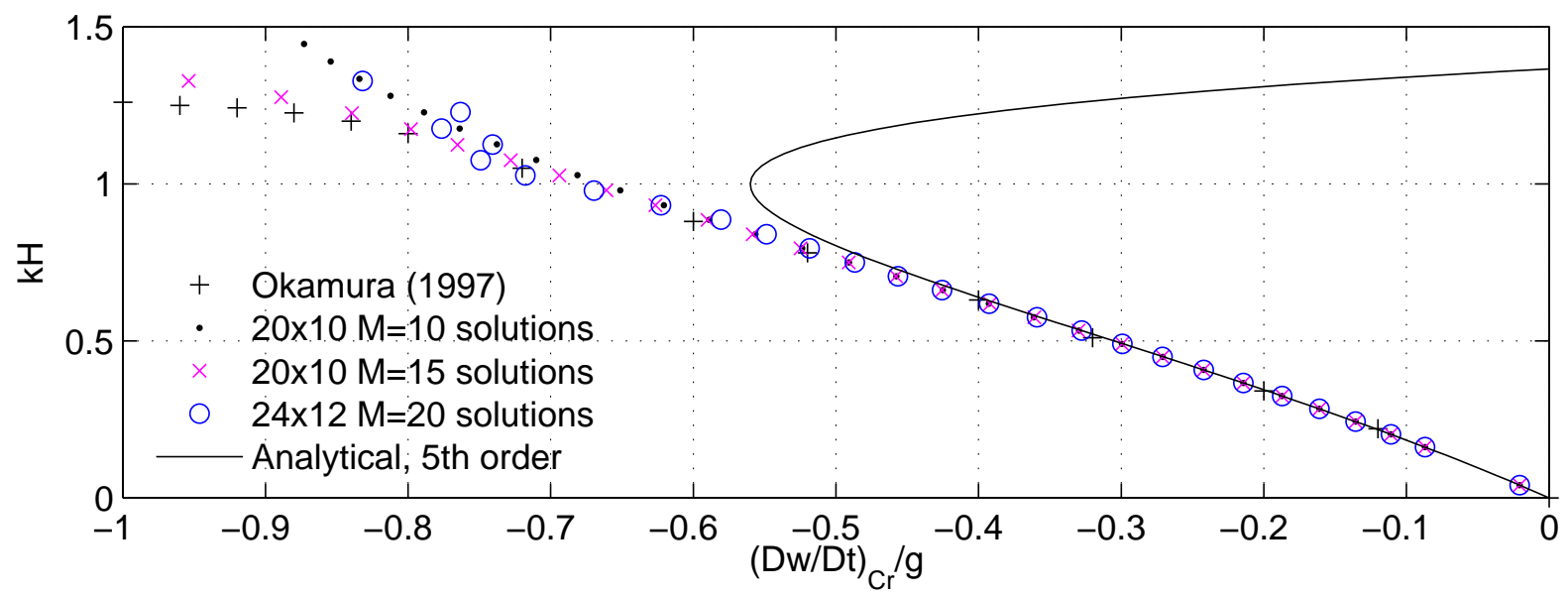

Figure 12: Progression to Limit Wave in Deep Water.

frequently adopted (Aoki, 1980; Schwartz and Whitney, 1981; Rottman, 1982; Mercer and Roberts, 1992; Okamura, 2003) in discussions of limit standing waves, mostly in deep water.

Mercer and Roberts (1992, Figure 2) and Okamura (1997, 2003, Figure 6) provide numerical predictions in deep water that reach almost to the limit wave as defined by Equation 35. It is not clear that the Mercer and Roberts (1992) boundary integral solution is a legitimate standing wave solution. Periodicity in $x$ can be imposed through the lateral boundary conditions. The numerical boundary integral solution evolves in time from a specified initial water surface profile. This is a potential flow, and any non-periodic component of the initial conditions will remain within the solution domain as disturbance waves. These issues must be confirmed by numerical experiment, and there is no discussion of the initial conditions and no confirmation of periodicity in $t$. Similarly, concerns regarding recognition of the MWL constraint and the dispersion relationship in the Okamura (1997, 2003) numerical Stokes-approximation solutions were addressed above. Nevertheless, these appear to be the most plausible indications available of the approach to the limit wave, albeit in deep water. In both cases, the information provided is a plot of $\mathrm{kH} / 2$ as a function of $\left|(D w / D t)_{\mathrm{Cr}}\right| / g$. Both plots are visually very similar. The predicted trends were somewhat easiest to interpolate from Okamura (1997, Figure 6).

Analytical predictions, Stokes Approximation predictions from Figures 8a and c for $\omega^{2} h / g=4.02$ (deep water) at $M=15$ and the Okamura (1997) predictions are presented together in Figure 12. Also included are Stokes Approximation predictions at the same deep water $\omega^{2} h / g$ for $M=10$ and $M=20$. Agreement among theories is excellent for $\left|(D w / D t)_{\mathrm{Cr}}\right| \lesssim 0.5 g$, but the divergence at more extreme wave heights provides an excellent illustration of both the benefits and the limitations of the numerical Stokes Approximation solutions. The inadequacy of the analytical theory beyond moderate wave heights has been documented elsewhere (Sobey, 2009). The present numerical theory at $M=10$ extends the domain of validity to about $0.65 \mathrm{~g}$, and at $M=15$ to about $0.8 \mathrm{~g}$. Extending the 
truncation order to $M=20$ does not move the Stokes Approximation predictions any closer to $g$ (Equation 35). They are consistently less satisfactory, a result that can be traced to the approach of the expected higher-order $A_{j m}$ and $b_{j m}$ coefficients in magnitude to the convergence criteria.

While the limit wave is beyond the predictive capability of either the analytical theory or the hybrid analytical-numerical Stokes Approximation theory, trend extrapolation to $(D w / D t)_{\mathrm{Cr}}=-g$ might provide a viable prediction of limit wave conditions. The predictive trends in Figure 12 on approach to the Equation 35 limit demonstrate that this is almost certainly a flawed methodology. The analytical theory curves away high at about $-0.5 \mathrm{~g}$ from the Okamura (1997) data and doubles back on itself. The numerical $M=10$ predictions also begin to curve away high but now at about $-0.65 \mathrm{~g}$, a pattern followed by the $M=15$ predictions at about $-0.8 \mathrm{~g}$. The $M=20$ predictions become erratic beyond $-0.7 \mathrm{~g}$. If any of these trend curves are truncated before they become visually unacceptable, it is clear that trend extrapolation will predict limit wave conditions that are very much on the high side of the Okamura (1997) data.

These limits, specifically

$$
\left.\frac{D w}{D t}\right|_{\mathrm{Cr}} \geq \begin{cases}-0.65 g & \text { for } M=10 \\ -0.8 g & \text { for } M=15\end{cases}
$$

have been adopted to separate physically appropriate solutions from visually acceptable solutions. In the absence of independent guidance in other than deep water, Equation 36 has also been adopted for the full range of depths, from shallow to deep. In shallow and shallow transitional water however, achievable solutions do not reach as far as these limits (see Figure 10).

The Equation 36b definition of physically appropriate solutions was imposed on those solutions included in the Figure 11 data base.

\section{Limit of Viable Solutions}

While it is clear from the above discussions based on Figure 12 that limit wave predictions are beyond the reach of the present Stokes Approximation theory, viable solutions to the Equation 36 bounds do nevertheless describe quite extreme standing waves. Integral wave parameters will be identified as $H_{0.5}, k_{0.5},\left.\eta_{\text {Cr }}\right|_{0.5}, \ldots$ or $H_{0.65}, k_{0.65},\left.\eta_{\text {Cr }}\right|_{0.65}, \ldots$ or $H_{0.8}, k_{0.8}$, $\left.\eta_{\mathrm{Cr}}\right|_{0.8}, \ldots$ where they meet the

$$
\left.\frac{D w}{D t}\right|_{\mathrm{Cr}}=-0.5 g \quad \text { or }-0.65 g \quad \text { or }-0.8 g
$$

criterion respectively.

Estimation of the $H_{0.5}$ through $\left.\eta_{\text {Cr }}\right|_{0.8}$ is possible from the Figure 11 data base, through interpolation and/or extrapolation. For depths from $100 \mathrm{~m}$ down to $8 \mathrm{~m}$, cubic spline interpolation was possible within the Figure 11 data base; Figure $13 \mathrm{a}$ at $h=40 \mathrm{~m}$ is an 


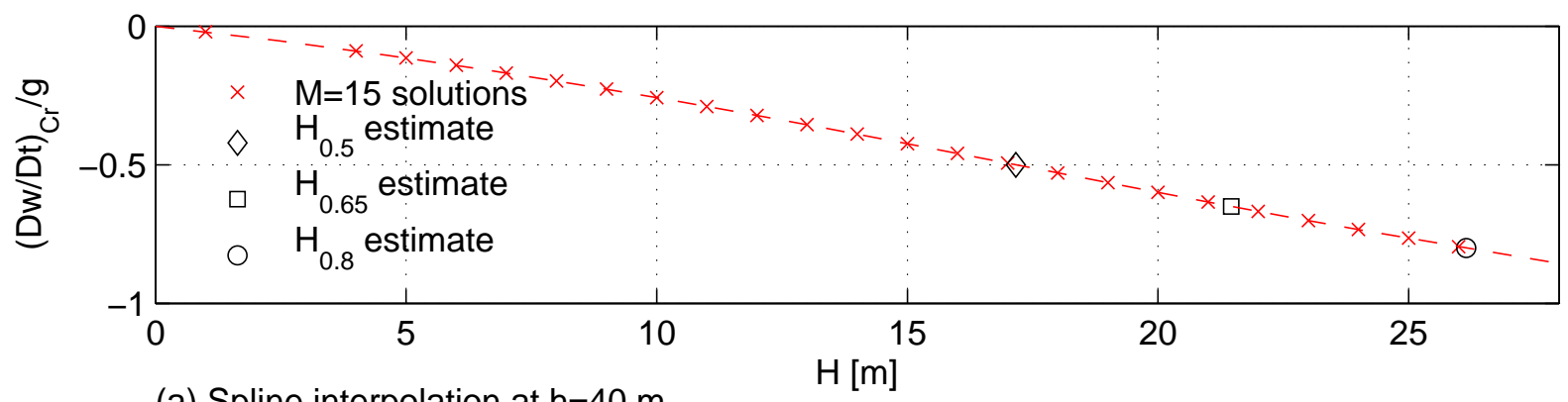

(a) Spline interpolation at $\mathrm{h}=40 \mathrm{~m}$.

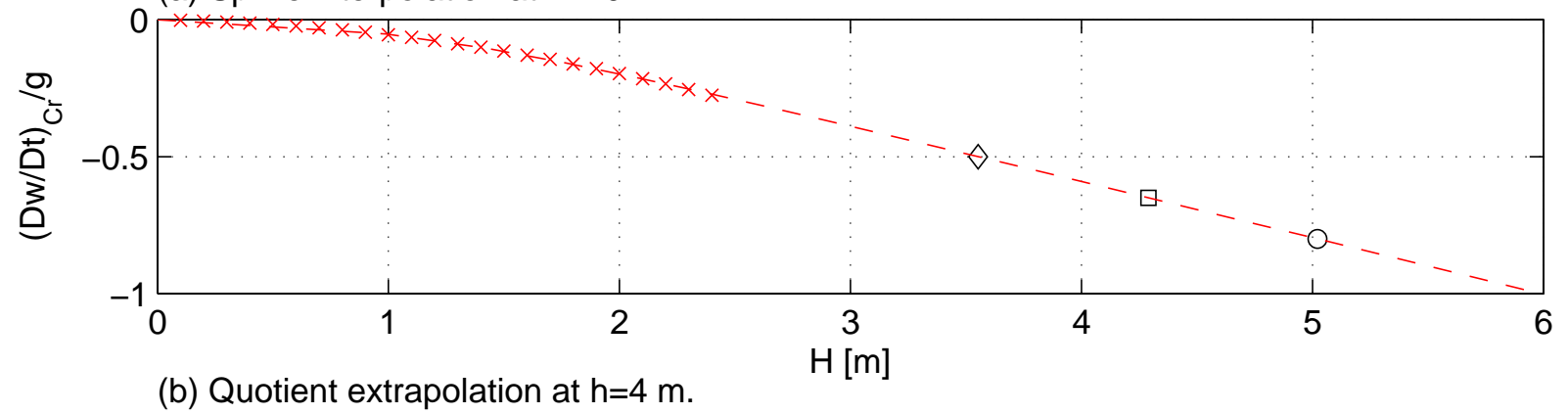

Figure 13: Viable Solution Estimation.

example. At $7 \mathrm{~m}$, spline interpolation was possible for the 0.50 and 0.65 estimates. Spline extrapolation for the 0.80 estimates seemed visually satisfactory. At $6 \mathrm{~m}$, solutions extended just beyond $D w / D t=-0.5 \mathrm{~g}$. For depths at $5 \mathrm{~m}$ and below, data base solutions did not even extend this far. At these smaller depths, polynomial quotient extrapolation, following

$$
y=\frac{a+b x+c x^{2}}{1+d x}
$$

was visually expedient. The parameters $a$ through $d$ were least-squares estimated from the appropriate $(x, y)$ data pairs, respectively $(D w / D t, H),(H, k)$ and $\left(H, \eta_{\mathrm{Cr}} / H\right)$, in the data base. Figure $13 \mathrm{~b}$ at $h=4 \mathrm{~m}$ is an example of polynomial quotient extrapolation.

These interpolation/extrapolation estimates are collected in Figure 14 for $H$ and in Figures 15 for $k$ and $\eta_{\mathrm{Cr}} / H$. Spline interpolation estimates are joined by a solid line, polynomial quotient extrapolation estimates by a dashed line. Non-dimensional scaling has been adopted to optimally identify the response patterns. The depths had been scaled as $\omega^{2} h / g$, following Equation 27 and Figure 11.

The wave height parameters $H_{0.5}, H_{0.65}$ and $H_{0.8}$ in Figure 14 roughly follow the pattern established for limit progressive waves, as anticipated in Figure 11 and confirmed in Figure 14 by the inclusion of the Equation 27 prediction for the limit progressive wave. Even in deep water, $H_{0.8}$ is at or above the limit progressive waves. Though the limit standing wave is not predicted by the present Stokes Approximation theory, the limit standing wave is clearly larger than the limit progressive wave under all conditions, the difference becoming 


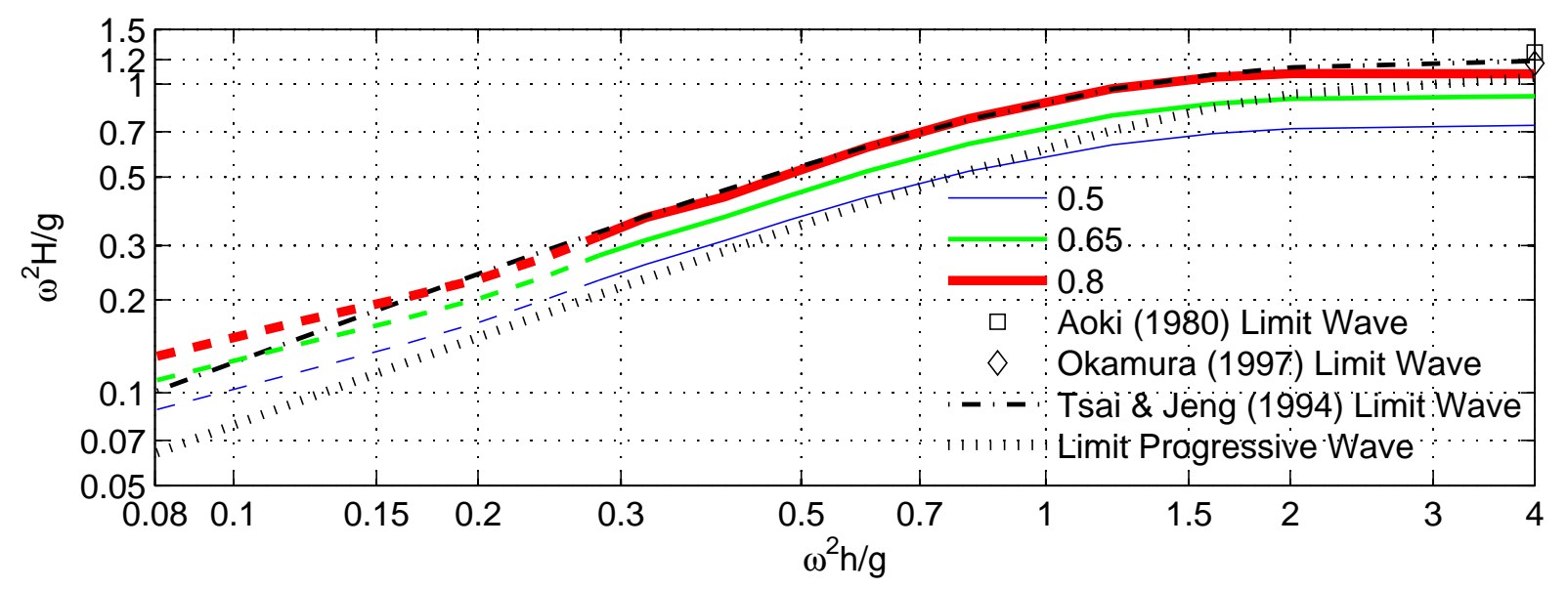

Figure 14: Extreme Heights Summary.

greater in shallower water. In shallower water, even $H_{0.5}$ exceeds the limit progressive wave. $H_{0.5}, H_{0.65}$ and $H_{0.8}$ follow a very similar pattern from deep to shallow water. The apparent almost uniform spacing follows from the roughly linear variation of $D w / D t$ with $H$; see, for example, Figures 8a through 10a.

Literature confirmation of Figure 14 is rather sparse, and entirely focussed on limit standing wave estimates. In all cases, the predictions are based on

(1) A theoretical model of the standing wave kinematics, and

(2) Extrapolation of evolution of $(D w / D t)_{\mathrm{Cr}}$ to $-g$ as $H$ increases

Of the field kinematics, prediction of the acceleration components is especially challenging. Using the present Stokes Approximation theory as an example, the $j m$ multiplier in the predictive equation for $\partial w / \partial t$ in Appendix B identifies an increasing influence for higher order and more strongly nonlinear contributions. The fidelity of the theoretical model is crucial, and this is emphasised in Figures 8c through 10c where the Sobey (2009) fifth-order analytical predictions for $(D w / D t)_{\mathrm{Cr}}$ become physically inappropriate (reach a minimum) significantly before $-g$.

The Aoki (1980) deep water theoretical model is analytical to eighth order, correcting an error at fifth order in Penney and Price (1952). It is recognised that the predictive equation for $(D w / D t)_{\mathrm{Cr}}$ is not valid at the crest, and a Shanks transformation provides the limit prediction $\eta_{\mathrm{Cr}} / L=0.138$. There is no detail of the evolution of $(D w / D t)_{\mathrm{Cr}}$ with $H$ with or without the Shanks transformation. Following the analytical experience at fifth order (Figure 8c), a minimum in the evolution is expected. Schwartz and Whitney (1981) and Rottman (1982) provide quite similar analyses. The Mercer and Roberts (1992) and Okamura $(1997,2003)$ contributions (see earlier discussion leading to Figure 12) provide deep water limit predictions of $k H / 2=0.62$ and 0.63 respectively. 

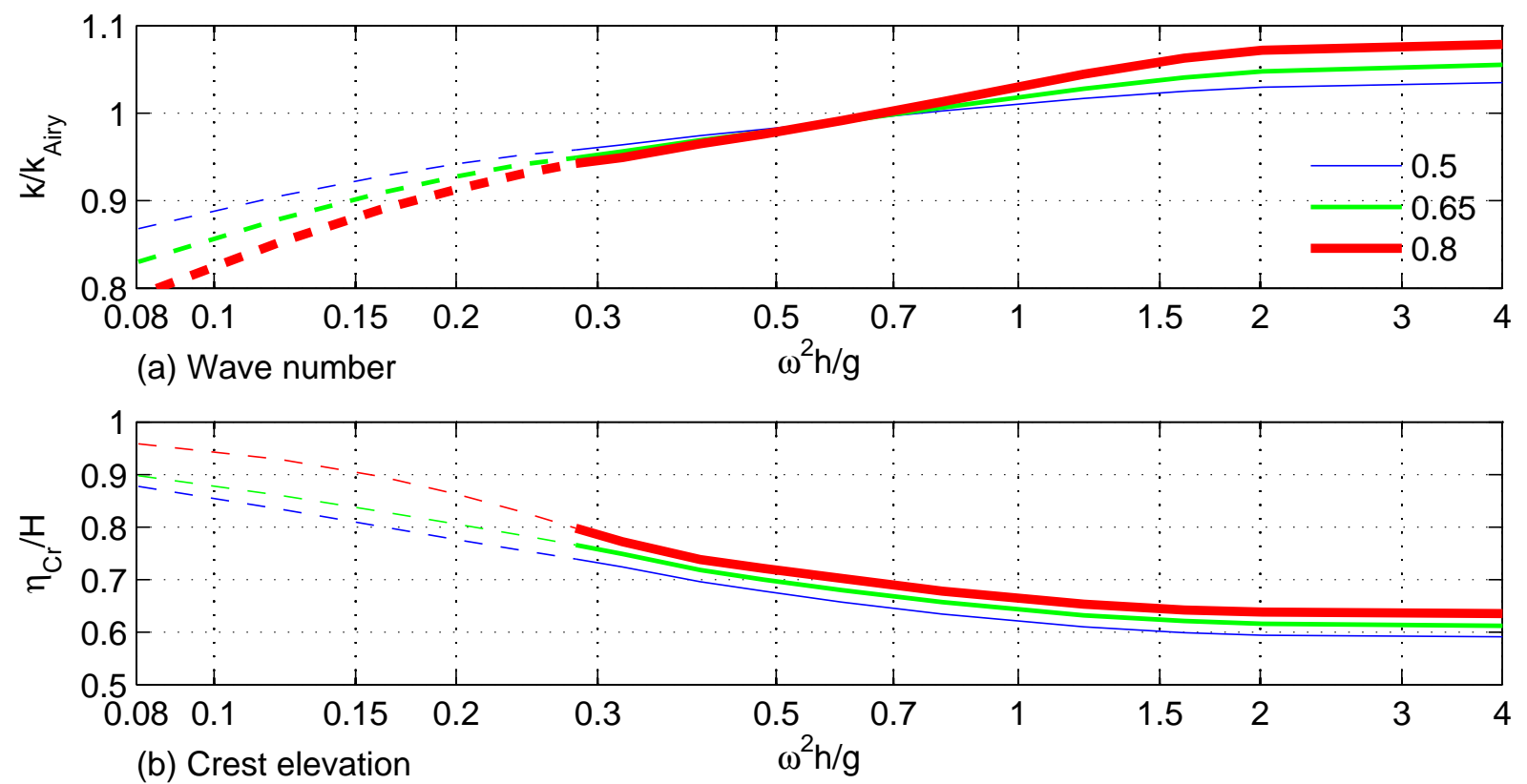

Figure 15: Wave Number and Crest Elevation at Extreme Heights.

Tsai and Jeng (1994, Figure 13 and Equation 22) provide predictions of the limit wave steepness at finite depths, curve-fitted as $H / L=0.204 \tanh (k h)$. Questions regarding the Tsai and Jeng (1994) algorithm were raised at the end of $\S 3$ Numerical Solution.

Each of the Aoki (1980), Okamura (1997, 2003) and Tsai and Jeng (1994) limit height predictions require some interpretation to present in the Figure 14 format. Specifically the limit wave number and wave frequency are not given, and also the crest elevation for the Aoki (1980) result. They have been translated to the Figure 14 format using $k_{0.8}$ and $\left.\eta_{\mathrm{Cr}}\right|_{0.8}$ from the present data base of solutions.

The Aoki (1980) and Okamura (1997, 2003) predictions for the limit standing wave height in deep water exceed $H_{0.8}$ as anticipated in Figure 12 and the associated discussion. The trend of the Tsai and Jeng (1994) prediction for the limit wave at finite depths has order of magnitude agreement with $H_{0.8}$ throughout, but it is not a sufficiently secure result to suggest any further significance.

The wave number parameters $k_{0.5}, k_{0.65}$ and $k_{0.8}$ in Figure 15 a generally follow the pattern established for progressive waves, namely a horizontal asymptote in deep water somewhat above the Airy asymptote of $\omega^{2} / g$ and a steepish climb to rather larger values in shallow water. This general trend is included in the linear dispersion relationship, Equation 29. The wave number parameters $k_{0.5}, k_{0.65}$ and $k_{0.8}$ have been scaled in Figure 15 a by $k_{\text {Airy }}$, the wave number predicted by Equation 29 . This has the benefit of focussing attention on non-linear influences on standing waves. Nonlinearity increases with wave height. In deep water, there are increases to about $8 \%$ above $k_{\text {Airy }}$. This trend is reversed as the water depth shallows, toward a deficit of order $20 \%$ below $k_{\text {Airy }}$ in shallow water. 
Finally, Figure $15 \mathrm{~b}$ shows the evolution of $\eta_{\mathrm{Cr}} / H$ with dimensionless depth. While polynomial quotient extrapolation seemed visually appropriate for both wave height (Figure 14) and wave number (Figure 14a) in shallow water, it completely failed for the crest elevation, providing extrapolations that reversed the upward trend as the water shallowed. These were discarded. Fortunately however, the extreme shallow water limit will be a standing solitary waves where the entire wave is above the mean water level, so that $\eta_{\mathrm{Cr}} / H \rightarrow 1$ at $h \rightarrow 0$. This limit will be appropriate for $\eta_{\mathrm{Cr}} /\left.H\right|_{0.5}, \eta_{\mathrm{Cr}} /\left.H\right|_{0.65}$ and $\eta_{\mathrm{Cr}} /\left.H\right|_{0.8}$. This additional data point at $h=0$ avoids extrapolation in shallow water. Together with the secure (solid line) estimates of $\eta_{\mathrm{Cr}} / H$ in transitional and deep water, cubic spline interpolation provides reasonable estimates for $\eta_{\mathrm{Cr}} /\left.H\right|_{0.5}, \eta_{\mathrm{Cr}} /\left.H\right|_{0.65}$ and $\eta_{\mathrm{Cr}} /\left.H\right|_{0.8}$ in shallow water. These are the dashed lines in Figure 14b. The response patterns in Figure 14b is entirely as expected. The crest super-elevation increases above 0.5 as the wave height increases, with the super-elevation increasing as the water shallows.

\section{Conclusions}

A hybrid analytical-numerical Stokes Approximation algorithm for standing waves over a very wide range of wave heights and water depths is outlined in $\S 2$ and $\S 3$.

The formulation $(\S 2)$ is analytical in part, exactly satisfying the field equation (Equation 1 ) and the periodic lateral boundary conditions (Equation 5) for all $x, z$, and $t$, the bottom boundary condition (Equation 2) for all $x$ and $t$, and also the invariant MWL constraint (Equation 7). The balance of the problem formulation, namely the specification of wave height (Equation 6) and the free surface boundary conditions (Equations 3 and 4), is completed numerically.

The details and challenges of the numerical solution are described in $\S 3$. The given information is wave height $H$, water depth $h$, wave period $T$, and mass density $\rho$. The unknowns of the numerical solution are the wave number $k$, Bernoulli constant $\bar{B}$, the $A_{j m}$ coefficients in Equation 11 for $\phi(x, z, t)$, and the $b_{j m}$ coefficients in Equation 12 for $\eta(x, t)$.

Available equations are provided by specification of wave height (Equation 14) and by the kinematic free surface boundary condition (Equation 15) and the dynamic free surface boundary condition (Equation 16) at distributed water surface nodes. These are nonlinear algebraic equations, solved as a problem in nonlinear optimisation by the trust region method, based on the Equation 19 objective function.

Given the numerical solution, $\S 4$ and Appendix B provide the predictive equations for the balance of the kinematics.

Confirmation of the Stokes Approximation method and its code implementation is provided in $\S 5$ by comparison with an existing fifth-order analytical theory. There is excellent predictive agreement in Figure 2 for a deep water wave of moderate height that is within the validity range of the analytical theory. The impact of increasing truncation order is explored in Figures 3 through 5. Truncation order $M$ evolves from little influence for moderate waves in deep water (Figure 3) through a small but important influence for extreme waves in deep water (Figure 4) to a very significant influence in shallow water 
(Figure 5). The Stokes Approximation theory is shown in Figure 6 to provide credible kinematic predictions at $M=10$ for a shallow wave of moderate height, in a context where the analytical theory has no credibility.

The clear failure of the analytical theory (e.g. Figures 5 and 6 ) when pushed beyond its region of validity raises the issue of identification of a credible numerical solution. This is addressed in $\S 6$. Three criteria are established. These proved useful in identifying visually successful numerical solutions.

Advantage is taken, in $\S 7$, of the enhanced credibility of the numerical theory in exploring response patterns in steepening waves from deep to shallow water. Figures 8 through 10 explore the solution evolution with increasing wave height in deep, transitional and shallow water respectively. Attention is directed to the wave number, the crest elevation and the vertical acceleration at the crest. The predictions of the fifth-order analytical theory are included as a datum for comparison. In each case, it is clear that the numerical theory has extended the range of validity to rather larger wave heights. However Figures 8 and 9 suggest that visually successful solutions have perhaps extended beyond the limit wave.

The inability of the Stokes Approximation method to predict the limit wave is acknowledged in $\S 8$. It is also demonstrated (Figure 12) that extrapolation of valid Stokes Approximation solutions is not a viable prospect, as such extrapolation will certainly predict a limit wave height that is much too high.

The data base of numerical solutions (Figure 11) nonetheless does have the potential to predict some near-limit extreme wave indicators. Extremity levels identified as $H_{0.5}, k_{0.5}$, $\left.\eta_{\mathrm{Cr}}\right|_{0.5}$ through $H_{0.8}, k_{0.8},\left.\eta_{\mathrm{Cr}}\right|_{0.8}$ are defined where the vertical acceleration at the crest has reached $0.5,0.65$ and 0.8 of the gravitational acceleration $g$; at the limit wave, $(D w / D t)_{\mathrm{Cr}}=$ $-g$. In $\S 9$, the extremity levels $H_{0.5}$ through $\left.\eta_{\mathrm{Cr}}\right|_{0.8}$ are predicted by interpolation and extrapolation from the Figure 11 data base. These extremity level predictions are presented non-dimensionally in Figure 14 for wave height $\omega^{2} \mathrm{H} / \mathrm{g}$ over the complete range of depths $\omega^{2} h / g$, and in Figures 15 for wave number $k / k_{\text {Airy }}$ and relative crest elevation $\eta_{\mathrm{Cr}} / H$ also over the complete range of depths $\omega^{2} h / g$. The more secure predictions involving interpolation are identified by the solid lines, and the less secure predictions involving extrapolation by the dashed lines.

\section{Acknowledgements}

The advice and assistance of Stuart Archibald in multi-core processing and OpenMP coding contributed significantly to the timely completion of this research.

\section{A Alternative Problem Formulation}

This appendix details the adjustments to the $\S 3$ Numerical Solution algorithm required by the choice of wave length rather that wave period among the given information.

(1) In the given information defining a standing wave problem, $L$ replaces $T$ in item (iii). 
(2) In the unknowns of the Stokes approximation problem, $\omega$ replaces $k$ in item (i).

(3) The number of unknowns remains as specified in Equation 13.

(4) The equations available remain unchanged as Equations 14, 15 and 16, except that the left hand sides become $f\left(\omega, \bar{B}, A_{j m}, b_{j m}\right)$ rather than $f\left(k, \bar{B}, A_{j m}, b_{j m}\right)$.

(5) The least squares objective function remains Equation 19, except that the left hand sides becomes $\mathrm{O}\left(\omega, \bar{B}, A_{j m}, b_{j m}\right)$ rather than $\mathrm{O}\left(k, \bar{B}, A_{j m}, b_{j m}\right)$.

(6) The length and time scales, Equation 20, become

$$
\mathbb{L}=1 / k, \quad \mathbb{T}=(g k)^{-1 / 2}
$$

(7) The initial solution estimate strategies remain unchanged, except that $S(H ; h, T, \rho, M)=$ $\left[k, \bar{B}, A_{j m}, b_{j m}\right]$ becomes $S(H ; h, L, \rho, M)=\left[\omega, \bar{B}, A_{j m}, b_{j m}\right]$.

(8) The Solution Kinematics remain Equations 11, 24, 25 and 26, together with Appendix B.

\section{B Supplementary Equations for Kinematics}

$$
\begin{aligned}
\phi(x, z, t) & =\sum_{j=0}^{M} \sum_{m=0}^{M} A_{j m} \frac{\cosh j k(h+z)}{\cosh j k h} \cos j k x \sin m \omega t \\
\frac{\partial \phi}{\partial t} & =\omega \sum_{j=0}^{M} \sum_{m=0}^{M} m A_{j m} \frac{\cosh j k(h+z)}{\cosh j k h} \cos j k x \cos m \omega t \\
\eta(x, t) & =\sum_{j=0}^{M} \sum_{m=0}^{M} b_{j m} \cos j k x \cos m \omega t \\
\frac{\partial \eta}{\partial t} & =-\omega \sum_{j=0}^{M} \sum_{m=0}^{M} m b_{j m} \cos j k x \sin m \omega t \\
\frac{\partial \eta}{\partial x} & =-k \sum_{j=0}^{M} \sum_{m=0}^{M} j b_{j m} \sin j k x \cos m \omega t
\end{aligned}
$$




$$
\begin{aligned}
u(x, z, t) & =\frac{\partial \phi}{\partial x}=-k \sum_{j=0}^{M} \sum_{m=0}^{M} j A_{j m} \frac{\cosh j k(h+z)}{\cosh j k h} \sin j k x \sin m \omega t \\
\frac{\partial u}{\partial t} & =-k \omega \sum_{j=0}^{M} \sum_{m=0}^{M} j m A_{j m} \frac{\cosh j k(h+z)}{\cosh j k h} \sin j k x \cos m \omega t \\
\frac{\partial u}{\partial x} & =-k^{2} \sum_{j=0}^{M} \sum_{m=0}^{M} j^{2} A_{j m} \frac{\cosh j k(h+z)}{\cosh j k h} \cos j k x \sin m \omega t \\
\frac{\partial u}{\partial z} & =-k^{2} \sum_{j=0}^{M} \sum_{m=0}^{M} j^{2} A_{j m} \frac{\sinh j k(h+z)}{\cosh j k h} \sin j k x \sin m \omega t \\
w(x, z, t) & =\frac{\partial \phi}{\partial z}=k \sum_{j=0}^{M} \sum_{m=0}^{M} j A_{j m} \frac{\sinh j k(h+z)}{\cosh j k h} \cos j k x \sin m \omega t \\
\frac{\partial w}{\partial t} & =k \omega \sum_{j=0}^{M} \sum_{m=0}^{M} j m A_{j m} \frac{\sinh j k(h+z)}{\cosh j k h} \cos j k x \cos m \omega t \\
\frac{\partial w}{\partial x} & =-k^{2} \sum_{j=0}^{M} \sum_{m=0}^{M} j^{2} A_{j m} \frac{\sinh j k(h+z)}{\cosh j k h} \sin j k x \sin m \omega t \\
\frac{\partial w}{\partial z} & =k^{2} \sum_{j=0}^{M} \sum_{m=0}^{M} j^{2} A_{j m} \frac{\cosh j k(h+z)}{\cosh j k h} \cos j k x \sin m \omega t
\end{aligned}
$$

\section{References}

Aoki, H. (1980). Higher order calculation of finite periodic standing gravity waves by means of the computer. Journal of Physical Society of Japan 49, 1598-1606.

Conn, A. R., N. I. M. Gould, and P. L. Toint (2000). Trust-Region Methods. MPS/SIAM Series on Optimization. Philadelphia, PA: SIAM Society for Industrial \& Applied Mathematics.

Goda, Y. (1967). The fourth order approximation to the pressure of standing waves. Coastal Engineering in Japan 10, 1-11.

Hsu, J. R. C., Y. Tsuchiya, and R. Silvester (1979). Third-order approximation to shortcrested waves. Journal of Fluid Mechanics 90, 179-196.

Mercer, G. N. and A. J. Roberts (1992). Standing waves in deep water: Their stability and extreme form. Physics of Fluids A 4, 259-269. 
Okamura, M. (1997). The profile of large amplitude standing waves (in Japanese). Kyoto University Research Information Repository, Citation 993: 134-143. URL: http://hdl.handle.net/2433/61179.

Okamura, M. (2003). Standing gravity waves of large amplitude in deep water. Wave Motion 37, 173-182.

Penney, W. G. and A. T. Price (1952). Part II. Finite periodic stationary gravity waves in a perfect fluid. Philosophical Transactions, Royal Society, London (A) 244, 254-284.

Press, W. H., S. A. Teukolsky, W. T. Vetterling, and B. P. Flannery (1992). Numerical Recipes in FORTRAN: The Art of Scientific Computing (2 ed.). Cambridge University Press, Cambridge.

Rienecker, M. M. and J. D. Fenton (1981). A Fourier approximation method for steady water waves. Journal of Fluid Mechanics 104, 119-137.

Rottman, J. W. (1982). Steep standing waves at a fluid interface. Journal of Fluid Mechanics 124, 283-306.

Schwartz, L. W. and A. K. Whitney (1981). A semi-analytical solution for nonlinear standing waves in deep water. Journal of Fluid Mechanics 10\%, 147-171.

Sobey, R. J. (1989). Variations on Fourier wave theory. International Journal for Numerical Methods in Fluids 9, 1453-1467.

Sobey, R. J. (2009). Analytical solutions for steep standing waves. Engineering and Computational Mechanics 162(EM4), 185-197.

Sobey, R. J. (2012). Steep progressive waves in deep and shallow water. Engineering and Computational Mechanics ?,?-? In Press; accepted May 2010.

Tadjbakhsh, I. and J. B. Keller (1960). Standing surface waves of finite amplitude. Journal of Fluid Mechanics 8, 442-451.

Tsai, C.-P. and D.-S. Jeng (1994). Numerical Fourier solutions of standing waves in finite water depth. Applied Ocean Research 16, 185-193.

Vanden-Broeck, J.-M. and L. W. Schwartz (1981). Numerical computation of steep gravity waves in shallow water. Physics of Fluids 24, 812-815.

Williams, J. M. (1985). Tables of Progressive Gravity Waves. Pitman, Boston. 